\title{
Multi-contrast anatomical subcortical structures parcellation
}

\author{
Pierre-Louis Bazin ${ }^{1,2 \star}$, Anneke Alkemade ${ }^{1}$, Martijn J Mulder ${ }^{1,3}$, Amanda G Henry ${ }^{4}$, \\ Birte U Forstmann ${ }^{1}$
}

${ }^{1}$ Integrative Model-based Cognitive Neuroscience research unit, University of Amsterdam, Amsterdam, Netherlands; ${ }^{2}$ Max-Planck Institute for Human Cognitive and Brain Sciences, Leipzig, Germany; ${ }^{3}$ Psychology Department, Utrecht University, Utrecht, Netherlands; ${ }^{4}$ Faculty of Archaeology, Leiden University, Leiden, Netherlands

\begin{abstract}
The human subcortex is comprised of more than 450 individual nuclei which lie deep in the brain. Due to their small size and close proximity, up until now only $7 \%$ have been depicted in standard MRI atlases. Thus, the human subcortex can largely be considered as terra incognita. Here, we present a new open-source parcellation algorithm to automatically map the subcortex. The new algorithm has been tested on 17 prominent subcortical structures based on a large quantitative MRI dataset at 7 Tesla. It has been carefully validated against expert human raters and previous methods, and can easily be extended to other subcortical structures and applied to any quantitative MRI dataset. In sum, we hope this novel parcellation algorithm will facilitate functional and structural neuroimaging research into small subcortical nuclei and help to chart terra incognita.
\end{abstract}

*For correspondence: pilou.bazin@uva.nl

Competing interests: The authors declare that no competing interests exist.

Funding: See page 19

Received: 28 May 2020

Accepted: 15 December 2020

Published: 16 December 2020

Reviewing editor: Timothy Verstynen, Carnegie Mellon University, United States

(c) Copyright Bazin et al. This article is distributed under the terms of the Creative Commons Attribution License, which permits unrestricted use and redistribution provided that the original author and source are credited.

\section{Introduction}

Subcortical brain structures are often neglected in neuroimaging studies due to their small size, limited inter-regional contrast, and weak signal-to-noise ratio in functional imaging (Forstmann et alo, 2016; Johansen-Berg, 2013). Yet, these small and diverse structures are prominent nodes in functional networks (Marquand et al., 2017; Ji et al., 2019), and they undergo pathological alterations already at early stages of neurodegenerative diseases (Andersen et al., 2014; Koshiyama et al., 2018). Deep brain stimulation surgery, originally performed to reduce motor symptoms in essential tremors, is now a promising therapeutic option in later stages of Parkinson's disease and movement disorders, as well as refractory psychiatric illnesses in obsessive-compulsive disorder, anorexia, or depression (Forstmann et al., 2017; Mosley et al., 2018). Evolutionary genetics even uncovered that in modern humans, Neanderthal-inherited alleles were preferentially down-regulated in subcortical and cerebellar regions compared to other brain regions (McCoy et al., 2017), suggesting these structures to be essential in making us specifically human.

Despite their importance, these areas are particularly difficult to image. Furthermore, the size, shape, and location of these brain regions changes with development and aging (Fjell et alo, 2013; Keuken et al., 2013; Yeatman et al., 2014; Herting et al., 2018). Experience-based plasticity continuously remodels myelin (Tardif et al., 2016; Hill et al., 2018; Turner, 2019), iron and other magnetic substances accumulate with age or pathology (Andersen et al., 2014; Zhang et al., 2018), both bringing changes in the MRI appearance of subcortical regions with diverse tissue characteristics (Draganski et al., 2011; Keuken et al., 2017).

Thus, mapping the structure and function of the subcortex is a major endeavor as well as a major challenge for human neuroscience. Extensive work available from animal brain models unfortunately does not translate in a straightforward way to human subcortical anatomy nor does it shed much light on its involvement in human cognition (Steiner and Tseng, 2017). Besides serious difficulties in 
obtaining adequate measures of subcortical neural activity in functional MRI (de Hollander et al., 2017; Miletić et al., 2020), atlases and techniques for labeling accurately and reliably individual subcortical structures have also been scarce (Frazier et al., 2005; Chakravarty et al., 2006; Ahsan et al., 2007; Yelnik et al., 2007; Qiu et al., 2010; Patenaude et al., 2011), typically labeling the thalamus, striatum (or its subdivision into caudate and putamen), and globus pallidus (internal and external segments combined), sometimes the amygdala. However, recent advances in anatomical MRI, combining multiple contrasts and/or quantitative MRI mapping and utilizing the higher resolution achievable with 7 Tesla (7T) and above have started to reduce the gap, each mapping a few additional structures or sub-structures, primarily the iron-rich substantia nigra, red nucleus and subthalamic nucleus (Keuken et al., 2013; Xiao et al., 2015; Visser et al., 2016a; Visser et al., 2016b; Wang et al., 2016; Makowski et al., 2018; Ewert et al., 2018; Iglesias et al., 2018; Pauli et al., 2018; Sitek et al., 2019). While these efforts generated valuable atlases, they do not yet enable to identify many subcortical structures in individual subjects. Manual delineation, on the other hand, requires extensive manual labor from highly trained experts which cannot be easily applied to large cohorts or clinical settings.

Here, we propose a new automated parcellation technique to identify and label 17 individual subcortical structures of varying size and composition in individual subjects, based on a large quantitative 7T MRI database (Alkemade et al., 2020), using quantitative maps of relaxation rates $\mathrm{R} 1$ and R2* (1/T1 and 1/T2*, respectively) and quantitative susceptibility maps (OSM) as anatomical contrasts. The algorithm, named Multi-contrast Anatomical Subcortical Structure Parcellation (MASSP), follows a Bayesian multi-object approach similar in essence to previous efforts (Fischl et al., 2002; Eugenio Iglesias et al., 2013; Visser et al., 2016a; Garzón et al., 2018), combining shape priors, intensity distribution models, spatial relationships, and global constraints. The main innovation of our approach is to explicitly estimate interfaces between subcortical structures based on a joint model derived from signed distance functions. Modeling interfaces in addition to the structure itself provides a rich basis to encode relationships and anatomical knowledge in shape and intensity priors. A voxel-wise Markovian diffusion regularizes the combined priors for each defined interface, lowering the imaging noise. Finally, the voxel-wise posteriors for the different structures and interfaces are further combined into global anatomical parcels by topology correction and region growing taking into account volumetric priors, which regularizes parcellation results further in smaller nuclei with low or heterogeneous contrast. To validate the results from this new method, in a thorough comparison with expert manual labeling, we show that the proposed method provides results very close from manual raters in many structures and exhibit reasonable bias across the adult lifespan. The method can easily be extended to new structures, can be applied to any quantitative MRI dataset and is available in Open Source as part of Nighres (Huntenburg et al., 2018), a neuroimage analysis package aimed at high-resolution neuroimaging.

\section{Results}

The MASSP parcellation method presented here has been trained to parcellate the following 17 structures: striatum (Str), thalamus (Tha), lateral, 3rd and 4th ventricles (LV, 3V, 4V), amygdala (Amg), globus pallidus internal segment (GPi) and external segment (GPe), SN, STN, red nucleus (RN), ventral tegmental area (VTA), fornix (fx), internal capsule (ic), periaqueductal gray (PAG), pedunculopontine nucleus (PPN), and claustrum $(\mathrm{Cl})$, see Figure 1. These structures include the most commonly defined subcortical regions (Str, Tha, Amg, LV), the main iron-rich nuclei (GPi, GPe, RN, SN, STN), as well as smaller, less studied areas (VTA, PAG, PPN, Cl), white matter structures (ic, $\mathrm{fx}$ ), and the central ventricles $(3 \mathrm{~V}, 4 \mathrm{~V})$.

MASSP uses a data set of ten expert delineations as a basis for its modeling. From the delineations, an atlas of interfaces between structures, shape skeletons, and interface intensity histograms are generated, and used as prior in a multiple-step non-iterative Bayesian algorithm, see Figure 2 and Materials and methods.

\section{Validation against manual delineations}

In a leave-one-out validation study comparing performance with the manual delineations, MASSP performed above $95 \%$ of the level of quality of the raters for Str, Tha, 4V, GPe, SN, RN, VTA, ic in terms of Dice overlap, the most stringent of the quality measures (see Figures 3 and 4 and Table 1). 

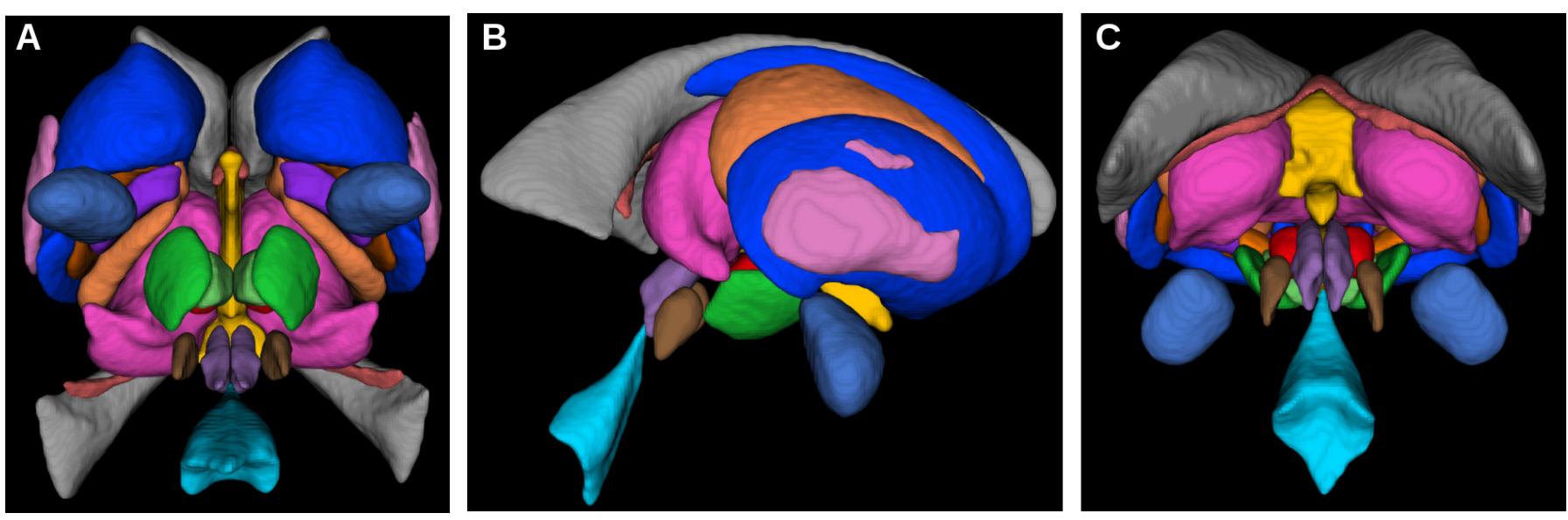

Figure 1. The 17 subcortical structures currently included in the parcellation algorithm in axial (A), sagittal (B), and coronal (C) views.

Several of the smaller structures have lower overlap ratios likely due to their smaller size (GPi, STN, PAG, PPN). Structures with an elongated shape $(f x, C l)$ remain challenging, due to the fact that small differences in location can substantially reduce overlap (Bazin et al., 2016). Despite these challenges, when comparing the dilated Dice scores, all structures were above $75 \%$ of overlap, with most reaching over $90 \%$ of the manual raters ability. Note that the Dice coefficient is very sensitive to size, as smaller structures will have lower overlap ratios for the same number of misclassified voxels. The dilated Dice coefficient is more representative of the variability regardless of size, as the smaller structures can reach high levels of overlap, both in manual and automated parcellations (see Table 1). The average surface distance confirms these results, showing values generally between one and two voxels of distance at a resolution of $0.7 \mathrm{~mm}$, except in the cases of Amg, LV, fx, PPN, and $\mathrm{Cl}$. These structures are generally more variable $(\mathrm{LV})$, elongated $\left(\mathrm{fx}_{\mathrm{x}}, \mathrm{Cl}\right)$, or have a particularly low contrast with neighboring regions (Amg, PPN).

\section{Comparison to other automated methods}

To provide a basis for comparison, we applied other freely available methods for subcortical structure parcellation to the same 10 subjects. MASSP performs similarly to or better than Freesurfer, FSL FIRST and a multi-atlas registration using ANTs (see Table 2). Multi-atlas registration provides high accuracy in most structures as well, but is biased toward under-estimating the size of smaller and elongated structures where overlap is systematically reduced across the individual atlas subjects. Multi-atlas registration is also quite computationally intensive when using multiple contrasts at high

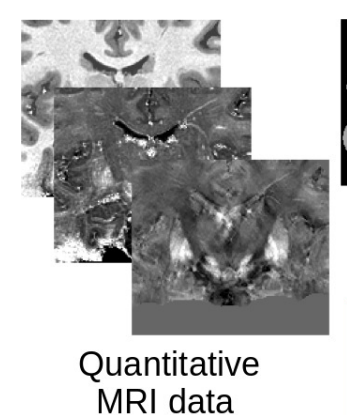

MRI data

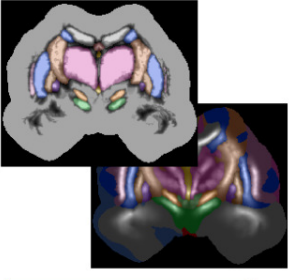

MASSP atlas co-registration

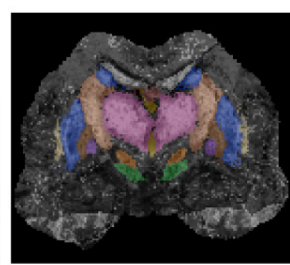

Priors and intensity joint probability

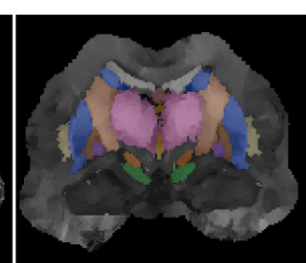

Markovian probability diffusion
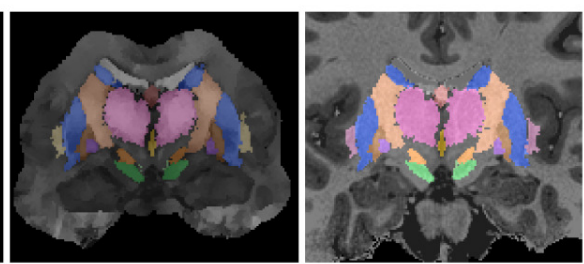

Anatomical region growing

Figure 2. The MASSP parcellation pipeline. Atlas priors for interfaces between structures are combined to the MRI data, regularized via probability diffusion and topology correction, and the final structure posteriors are jointly estimated by region growing. 


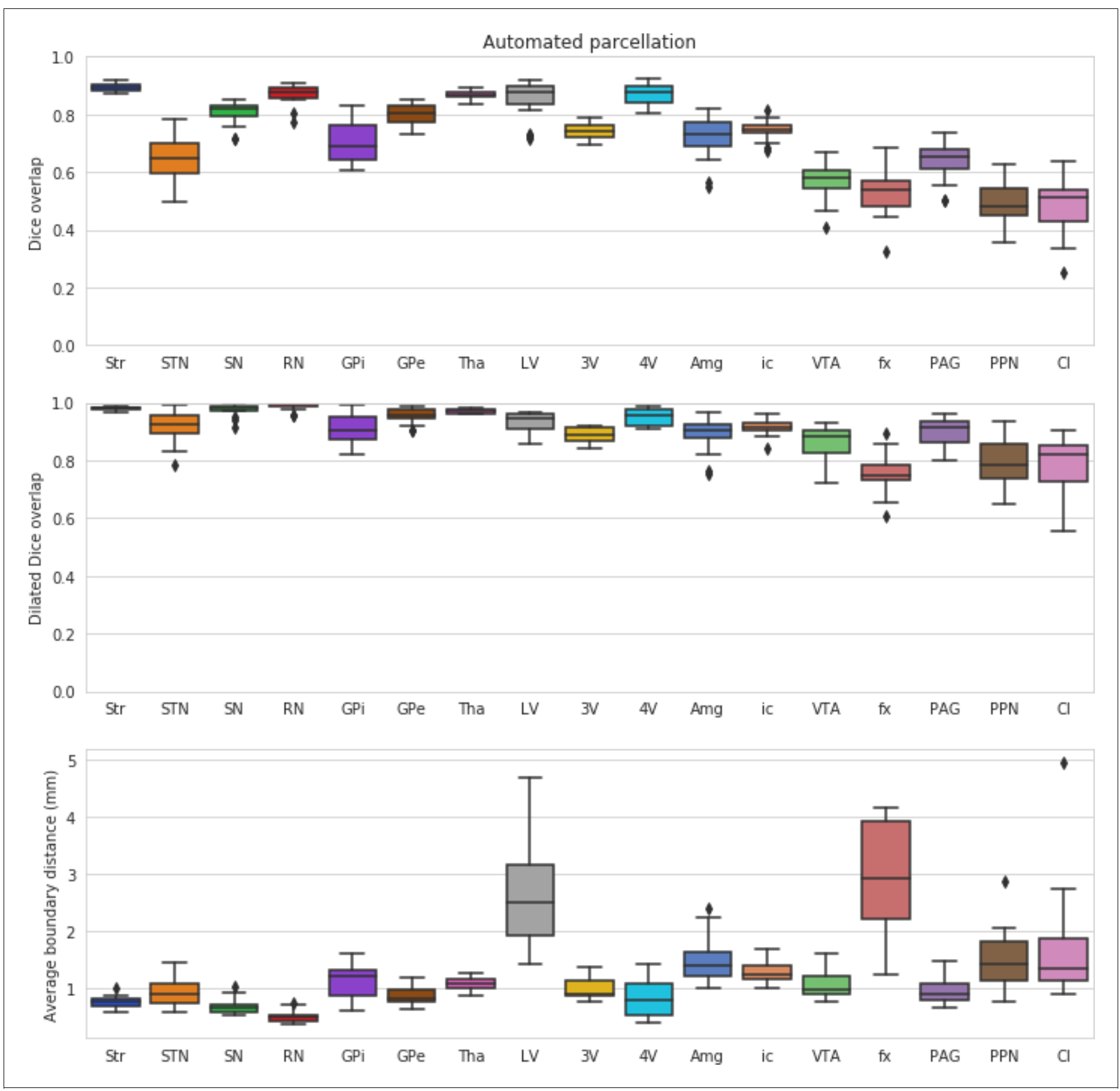

Figure 3. Leave-one-out validation of the structures parcellated by MASSP, compared to the human rater with most neuroanatomical expertise. Scores for the left and right side are computed separately and then combined into box-and-whisker plots.

resolution. Finally, MASSP provides many more structures than Freesurfer and FSL FIRST, and can be easily applied to new structures based on additional manual delineations.

\section{Application to new MRI contrasts}

Quantitative MRI has only become recently applicable in larger studies, thanks in part to the development of integrated multi-parameter sequences (Weiskopf et al., 2013; Caan et al., 2019). Many data sets, including large-scale open databases, use more common T1- and T2-weighted MRI. In 


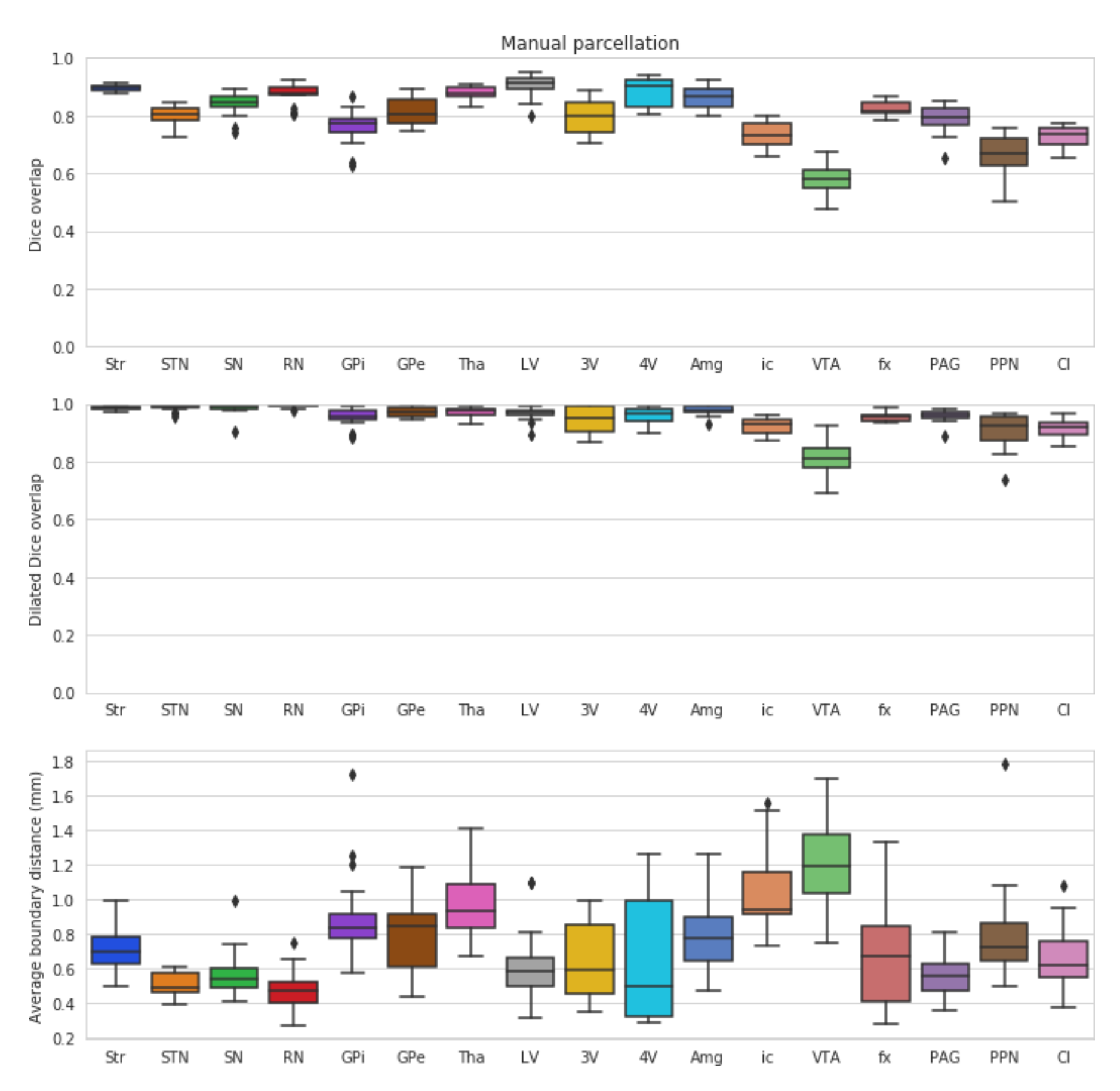

Figure 4. Inter-rater variability for the human expert raters. Scores for the left and right side are computed separately and then combined into boxand-whisker plots.

order to test the applicability of MASSP to such contrasts, we obtained the test-retest subset of the Human Connectome Project (HCP, Van Essen et al., 2013) and applied MASSP to the 45 pre-processed and skull-stripped T1- and T2-weighted images from each of the two test and retest sessions. While performing manual delineations on the new contrasts would be preferable, the model is already rich enough to provide stable parcellations. Test-retest reproducibility is similarly high for MASSP and Freesurfer, and are generally in agreement, see Figure 5 and Table 3. 
Table 1. Mean overlap and distance measures for the leave-one-out validation.

\begin{tabular}{|c|c|c|c|c|c|c|c|c|c|c|c|c|c|c|c|c|c|}
\hline & Str & STN & SN & RN & GPi & GPe & Tha & LV & $3 V$ & $4 V$ & Amg & ic & VTA & $f x$ & PAG & PPN & $\mathrm{Cl}$ \\
\hline \multicolumn{18}{|c|}{ Dice overlap } \\
\hline MASSP & 0.893 & 0.648 & 0.805 & 0.870 & 0.702 & 0.800 & 0.867 & 0.849 & 0.741 & 0.869 & 0.723 & 0.745 & 0.570 & 0.527 & 0.641 & 0.496 & 0.485 \\
\hline Manual & 0.897 & 0.800 & 0.841 & 0.875 & 0.762 & 0.813 & 0.877 & 0.907 & 0.797 & 0.882 & 0.866 & 0.732 & 0.574 & 0.823 & 0.791 & 0.665 & 0.727 \\
\hline Ratio & 0.995 & 0.811 & 0.957 & 0.996 & 0.925 & 0.987 & 0.989 & 0.936 & 0.936 & 0.988 & 0.836 & 1.020 & 0.994 & 0.641 & 0.814 & 0.754 & 0.664 \\
\hline \multicolumn{18}{|c|}{ Dilated overlap } \\
\hline MASSP & 0.982 & 0.919 & 0.977 & 0.991 & 0.909 & 0.956 & 0.970 & 0.929 & 0.890 & 0.951 & 0.891 & 0.915 & 0.863 & 0.756 & 0.897 & 0.795 & 0.789 \\
\hline Manual & 0.987 & 0.988 & 0.985 & 0.995 & 0.953 & 0.972 & 0.970 & 0.967 & 0.944 & 0.961 & 0.978 & 0.924 & 0.818 & 0.957 & 0.960 & 0.910 & 0.914 \\
\hline Ratio & 0.995 & 0.930 & 0.992 & 0.995 & 0.955 & 0.984 & 1.000 & 0.961 & 0.946 & 0.991 & 0.911 & 0.992 & 1.059 & 0.790 & 0.935 & 0.879 & 0.863 \\
\hline \multicolumn{18}{|c|}{ Average surface distance } \\
\hline MASSP & 0.750 & 0.911 & 0.676 & 0.491 & 1.140 & 0.863 & 1.058 & 2.690 & 0.994 & 0.817 & 1.476 & 1.275 & 1.074 & 2.950 & 0.955 & 1.484 & 1.685 \\
\hline Manual & 0.723 & 0.508 & 0.571 & 0.482 & 0.902 & 0.804 & 0.971 & 0.615 & 0.637 & 0.671 & 0.779 & 1.045 & 1.204 & 0.703 & 0.555 & 0.801 & 0.670 \\
\hline Ratio & 0.971 & 0.590 & 0.852 & 0.996 & 0.861 & 0.943 & 0.916 & 0.277 & 0.662 & 1.020 & 0.553 & 0.834 & 1.161 & 0.287 & 0.610 & 0.619 & 0.465 \\
\hline
\end{tabular}

\section{Biases due to atlas size}

A common concern of brain parcellation methods is the risk of biases, as they are typically built from a small number of manual delineations. Our data set is part of a large scale study of the subcortex, for which we obtained manual delineations of the STN, SN, RN, GPe, and GPi on 105 subjects over the adult lifespan (18-80 year old, see Alkemade et al., 2020 for details). First, we investigated the impact of atlas size. We randomly assigned half of the subjects from each decade to two groups, and built atlas priors from subsets of $3,5,8,10,12,15$, and 18 subjects from the first group. The subjects used in the atlas were taken randomly from each decade (18-30, 31-40, 41-50,51-60, 61-70, $71-80)$, so as to maximize the age range represented in each atlas. Atlases of increasing size were

Table 2. Comparison with multi-atlas registration, Freesurfer, and FSL FIRST.

\begin{tabular}{|c|c|c|c|c|c|c|c|c|c|c|c|c|c|c|c|c|c|}
\hline & Str & STN & SN & RN & GPi & GPe & Tha & LV & $3 V$ & $4 \mathrm{~V}$ & Amg & Ic & VTA & Fx & PAG & PPN & $\mathrm{Cl}$ \\
\hline \multicolumn{18}{|c|}{ Dice overlap } \\
\hline MASSP & 0.893 & 0.648 & 0.805 & 0.870 & 0.702 & 0.800 & 0.867 & 0.849 & 0.741 & 0.869 & 0.723 & 0.745 & 0.570 & 0.527 & 0.641 & 0.496 & 0.485 \\
\hline Multi-atlas & 0.855 & 0.662 & 0.760 & 0.820 & 0.742 & 0.796 & 0.859 & 0.734 & 0.660 & 0.691 & 0.761 & 0.718 & 0.626 & 0.478 & 0.674 & 0.539 & 0.398 \\
\hline Freesurfer & 0.876 & & & & 0.778 & & 0.838 & 0.858 & 0.430 & 0.769 & 0.692 & & & & & & \\
\hline FSL FIRST & 0.875 & & & & 0.813 & & 0.839 & & & & 0.653 & & & & & & \\
\hline \multicolumn{18}{|c|}{ Dilated overlap } \\
\hline MASSP & 0.982 & 0.919 & 0.977 & 0.991 & 0.909 & 0.956 & 0.970 & 0.929 & 0.890 & 0.951 & 0.891 & 0.915 & 0.863 & 0.756 & 0.897 & 0.795 & 0.789 \\
\hline Multi-atlas & 0.976 & 0.938 & 0.968 & 0.989 & 0.947 & 0.968 & 0.970 & 0.920 & 0.920 & 0.908 & 0.921 & 0.939 & 0.924 & 0.798 & 0.943 & 0.871 & 0.811 \\
\hline Freesurfer & 0.975 & & & & 0.922 & & 0.946 & 0.974 & 0.562 & 0.911 & 0.857 & & & & & & \\
\hline FSL FIRST & 0.976 & & & & 0.946 & & 0.950 & & & & 0.843 & & & & & & \\
\hline \multicolumn{18}{|c|}{ Average surface distance } \\
\hline MASSP & 0.750 & 0.911 & 0.676 & 0.491 & 1.140 & 0.863 & 1.058 & 2.690 & 0.994 & 0.817 & 1.476 & 1.275 & 1.074 & 2.950 & 0.955 & 1.484 & 1.685 \\
\hline Multi-atlas & 0.961 & 0.891 & 0.858 & 0.675 & 0.992 & 0.882 & 1.083 & 1.417 & 0.932 & 1.249 & 1.359 & 1.129 & 0.813 & 1.362 & 0.794 & 1.055 & 1.273 \\
\hline Freesurfer & 0.770 & & & & 1.211 & & 1.405 & 0.685 & 4.071 & 1.361 & 1.749 & & & & & & \\
\hline FSL FIRST & 0.867 & & & & 1.143 & & 1.675 & & & & 1.746 & & & & & & \\
\hline \multicolumn{18}{|c|}{ Volume bias } \\
\hline MASSP & 0.041 & 0.017 & -0.038 & 0.007 & 0.066 & 0.089 & 0.040 & 0.0470 & 0.121 & 0.047 & 0.078 & 0.183 & 0.032 & -0.016 & 0.026 & 0.009 & 0.023 \\
\hline Multi-atlas & 0.020 & -0.087 & -0.009 & 0.031 & 0.009 & 0.014 & 0.020 & -0.003 & -0.007 & -0.092 & -0.038 & 0.055 & -0.067 & -0.264 & -0.090 & -0.269 & -0.376 \\
\hline Freesurfer & 0.017 & & & & 0.087 & & 0.163 & 0.122 & -0.551 & 0.351 & 0.468 & & & & & & \\
\hline FSL FIRST & -0.100 & & & & -0.021 & & 0.165 & & & & -0.249 & & & & & & \\
\hline
\end{tabular}




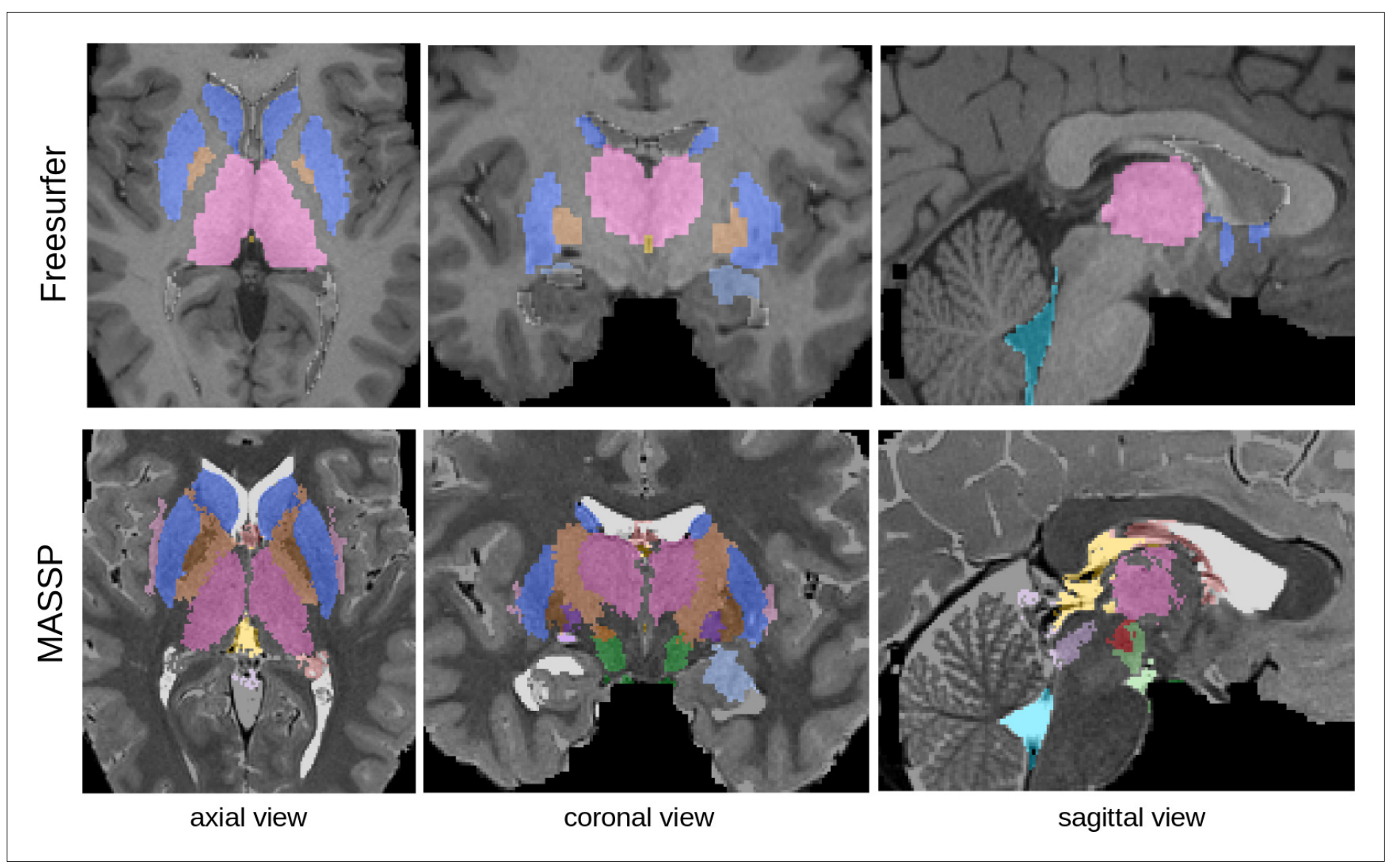

Figure 5. Parcellation with Freesurfer (top, on T1w image) and MASSP (bottom, on T2w image) on Human Connectome Project data. MASSP priors were not derived from the contrasts, but transferred via a spatial mapping of the quantitative MRI intensities from AHEAD subjects.

constructed by adding subjects to previous atlases, so that atlases of increasing complexity include all subjects from simpler atlases. Results applying these atlases to parcellate the second group are given in Figure 6. As in previous studies (Eugenio Iglesias et alo, 2013; Bazin and Pham, 2008), performance quickly stabilized with atlases of more than five subjects (no significant difference in

Table 3. Test-retest comparison with Freesurfer on Human Connectome Project data.

\begin{tabular}{|c|c|c|c|c|c|c|c|c|c|c|c|c|c|c|c|c|c|}
\hline & Str & STN & SN & RN & GPi & GPe & Tha & LV & $3 V$ & $4 V$ & Amg & ic & VTA & $f x$ & PAG & PPN & $\mathrm{Cl}$ \\
\hline \multicolumn{18}{|c|}{ Dice overlap } \\
\hline MASSP test-retest & 0.914 & 0.701 & 0.818 & 0.829 & 0.791 & 0.859 & 0.928 & 0.881 & 0.837 & 0.870 & 0.866 & 0.860 & 0.738 & 0.774 & 0.714 & 0.713 & 0.785 \\
\hline Freesurfer test-retest & 0.898 & & & & \multicolumn{2}{|c|}{0.770} & 0.919 & 0.894 & 0.842 & 0.849 & 0.852 & & & & & & \\
\hline MASSP - Freesurfer & 0.876 & & & & \multicolumn{2}{|c|}{0.778} & 0.838 & 0.858 & 0.430 & 0.769 & 0.692 & & & & & & \\
\hline \multicolumn{18}{|c|}{ Dilated overlap } \\
\hline MASSP test-retest & 0.987 & 0.939 & 0.977 & 0.978 & 0.963 & 0.977 & 0.990 & 0.980 & 0.979 & 0.986 & 0.981 & 0.973 & 0.969 & 0.961 & 0.965 & 0.972 & 0.966 \\
\hline Freesurfer test-retest & 0.986 & & & & \multicolumn{2}{|c|}{0.926} & 0.986 & 0.989 & 0.972 & 0.975 & 0.978 & & & & & & \\
\hline MASSP - Freesurfer & 0.954 & & & & \multicolumn{2}{|c|}{0.788} & 0.919 & 0.934 & 0.435 & 0.901 & 0.866 & & & & & & \\
\hline \multicolumn{18}{|c|}{ Average surface distance } \\
\hline MASSP test-retest & 0.513 & 0.528 & 0.467 & 0.461 & 0.532 & 0.508 & 0.488 & 0.509 & 0.391 & 0.419 & 0.533 & 0.536 & 0.431 & 0.464 & 0.428 & 0.402 & 0.433 \\
\hline Freesurfer test-retest & 0.876 & & & & \multicolumn{2}{|c|}{0.778} & 0.838 & 0.858 & 0.430 & 0.769 & 0.692 & & & & & & \\
\hline MASSP - Freesurfer & 0.976 & & & & \multicolumn{2}{|c|}{1.673} & 1.605 & 1.946 & 5.699 & 1.428 & 1.478 & & & & & & \\
\hline
\end{tabular}




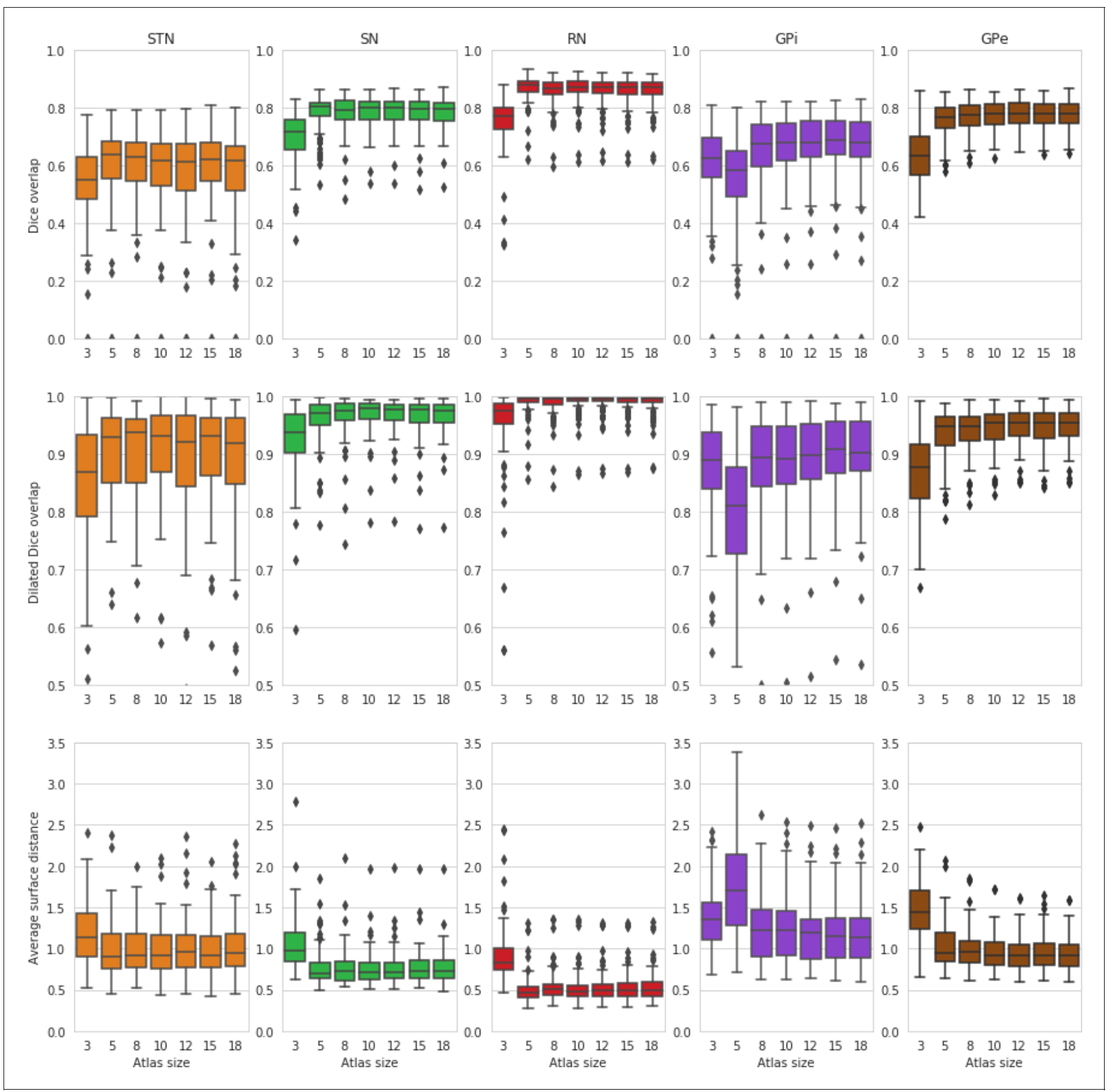

Figure 6. MASSP parcellation scores as a function of increasing number of subjects included in the atlas. Scores for the left and right side are computed separately and then combined into box-and-whisker plots.

Welch's t-tests between using 18 subjects or any subset of 8 or more for all structures and measures).

\section{Biases due to age differences}

To more specifically test the influence of age on parcellation accuracy, we defined again six age groups by decade and randomly selected 10 subjects from each group. Each set of subjects was used as priors for the five structures above, and applied to the other age groups. Results are 


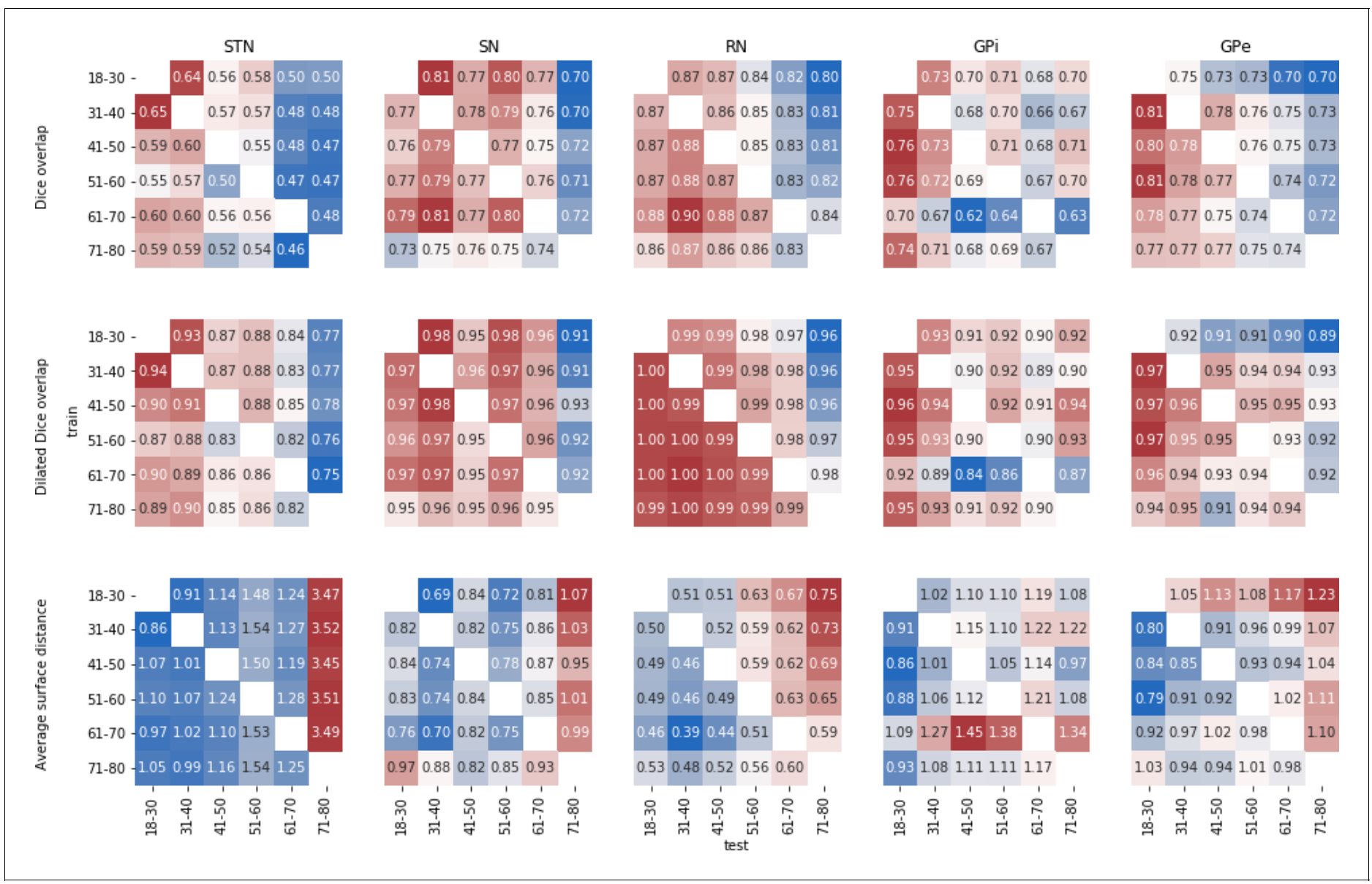

Figure 7. MASSP parcellation scores over the lifespan. Each matrix show the average Dice overlap (top), dilated Dice overlap (middle), and average surface distance (bottom) for using one age group as prior ('train') to parcellate another age group ('test').

summarized in Figure 7. Examining this age bias, we can see a decrease in performance when parcellating subjects in the range of 60 to 80 years of age. The choice of priors seem to have a limited impact, which varies across structures. In particular, using priors from a similar age group is not always beneficial.

\section{Bias on individual measures}

Finally, we investigated the impact of this decrease in performance in the estimation of anatomical quantities, see Figure 8. The bias did affect the morphometric measures of structure volume and thickness, but the effects on the local measure of thickness was reduced compared to the global measure of volume. Quantitative MRI averages were very stable even when age biases are present in the parcellations.

For reference, we report structure volumes, thickness, R1, R2* and OSM values estimated from the entire AHEAD cohort for different age groups, extending our previous work based on manual delineations on a different data set (Keuken et al., 2017; Forstmann et al., 2014). Results are given in Table 4, describing average volumes, thickness, and quantitative MRI parameters for young, middle-aged, and older subjects for the 17 subcortical structures.

\section{Discussion}

Our goal with the MASSP algorithm was to provide a fully automated method to delineate as many subcortical structures as possible on high-resolution structural MRI now available on 7T scanners. We 


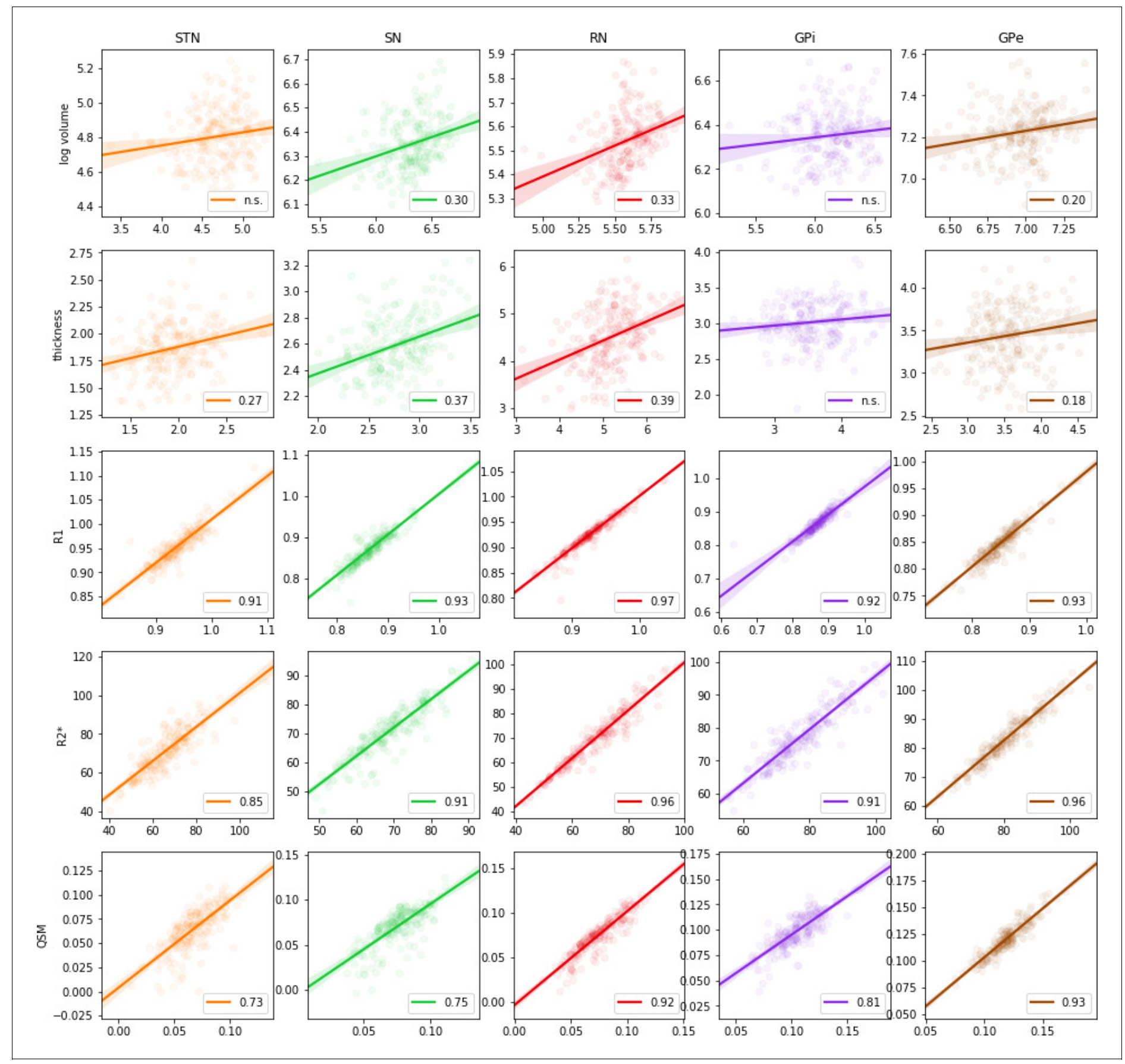

Figure 8. Regression of volume (log scale), structure thickness, R1, R2*, and QSM MRI parameters estimated using manual delineations versus MASSP automated parcellations. Circles show individual data points, linear regression is indicated by a straight line, and $95 \%$ confidence interval is given as the shaded area. Pearson correlation coefficients are indicated when significant ( $p$-value $<0.01)$.

modeled 17 distinct structures, taking into account location, shape, volume, and quantitative MRI contrasts to provide individual subject parcellations. Based on our results, we can be confident that the automated parcellation technique performs comparably to human experts, providing delineations within one or two voxels of the structure boundaries (dilated Dice overlap over $75 \%$ for all structures, including in aging groups). Results were nearly indistinguishable from expert delineations for eight major structures (Str, Tha, 4V, GPe, SN, RN, VTA, ic), and smaller structures retain high levels of overlap, comparable to trained human raters. This parcellation includes the most commonly defined structures (Str, Tha, SN, RN, STN) with overlap scores comparable to those previously 
Table 4. Mean volume and quantitative MRI values for each age group.

Age

\begin{tabular}{|c|c|c|c|c|c|c|c|c|c|c|c|c|c|c|c|c|c|}
\hline & Str & STN & SN & RN & GPi & GPe & Tha & LV & $3 V$ & $4 \mathrm{~V}$ & Amg & ic & VTA & $f x$ & PAG & PPN & $\mathrm{Cl}$ \\
\hline \multicolumn{18}{|c|}{ Volume $\left(\mathrm{mm}^{3}\right)$} \\
\hline $18-40$ & 10656 & 118 & 566 & 253 & 567 & 1366 & 7112 & 7524 & 1895 & 1391 & 1315 & 4335 & 254 & 1632 & 250 & 193 & 843 \\
\hline $41-60$ & 10572 & 124 & 583 & 256 & 586 & 1403 & 7492 & 8850 & 2024 & 1408 & 1363 & 4495 & 264 & 1808 & 255 & 195 & 830 \\
\hline $61-80$ & 10734 & 130 & 584 & 260 & 586 & 1397 & 7463 & 9142 & 2023 & 1407 & 1321 & 4407 & 272 & 1910 & 259 & 192 & 829 \\
\hline \multicolumn{18}{|c|}{ Thickness $(\mathrm{mm})$} \\
\hline $18-40$ & 5.94 & 1.89 & 2.55 & 4.64 & 3.09 & 3.56 & 8.31 & 4.27 & 2.77 & 4.03 & 4.81 & 4.06 & 1.69 & 2.06 & 1.78 & 1.92 & 1.79 \\
\hline $41-60$ & 5.47 & 1.86 & 2.66 & 4.58 & 2.96 & 3.41 & 8.28 & 5.08 & 2.95 & 3.89 & 4.85 & 4.19 & 1.76 & 1.96 & 1.84 & 1.86 & 1.80 \\
\hline $61-80$ & 5.22 & 1.83 & 2.60 & 4.11 & 2.92 & 3.22 & 8.28 & 4.90 & 3.18 & 4.06 & 4.73 & 4.19 & 1.80 & 1.97 & 1.90 & 1.95 & 1.82 \\
\hline \multicolumn{18}{|c|}{ qR1 $(H z)$} \\
\hline $18-40$ & 0.647 & 0.949 & 0.857 & 0.928 & 0.868 & 0.850 & 0.761 & 0.332 & 0.346 & 0.274 & 0.546 & 0.906 & 0.819 & 0.714 & 0.654 & 0.779 & 0.650 \\
\hline $41-60$ & 0.662 & 0.968 & 0.893 & 0.939 & 0.879 & 0.856 & 0.758 & 0.278 & 0.315 & 0.269 & 0.559 & 0.904 & 0.833 & 0.671 & 0.653 & 0.771 & 0.664 \\
\hline $61-80$ & 0.648 & 0.952 & 0.882 & 0.903 & 0.860 & 0.830 & 0.743 & 0.273 & 0.300 & 0.270 & 0.552 & 0.884 & 0.814 & 0.638 & 0.647 & 0.764 & 0.669 \\
\hline \multicolumn{18}{|c|}{ qR2* $(H z)$} \\
\hline $18-40$ & 43.8 & 67.1 & 67.8 & 63.2 & 75.2 & 79.6 & 38.1 & 14.7 & 18.9 & 9.0 & 25.5 & 36.8 & 39.2 & 37.4 & 25.9 & 32.7 & 32.6 \\
\hline $41-60$ & 50.4 & 74.1 & 74.1 & 77.1 & 80.2 & 87.9 & 40.3 & 8.4 & 12.4 & 11.7 & 28.1 & 38.7 & 42.8 & 37.4 & 28.0 & 33.4 & 36.9 \\
\hline $61-80$ & 51.8 & 77.0 & 72.5 & 73.8 & 77.8 & 87.0 & 40.1 & 8.5 & 10.2 & 12.0 & 30.1 & 39.6 & 52.6 & 35.7 & 28.4 & 34.2 & 35.4 \\
\hline \multicolumn{18}{|c|}{ QSM ( } \\
\hline $18-40$ & 0.0329 & 0.0609 & 0.0738 & 0.0717 & 0.1015 & 0.1150 & 0.0138 & 0.0130 & 0.0100 & 0.0279 & 0.0036 & -0.0234 & 0.0241 & 0.0079 & 0.0119 & 0.0135 & -0.0122 \\
\hline $41-60$ & 0.0400 & 0.0647 & 0.0713 & 0.0829 & 0.0984 & 0.1241 & 0.0134 & 0.0115 & 0.0025 & 0.0234 & 0.0085 & -0.0226 & 0.0201 & 0.0079 & 0.0089 & 0.0099 & -0.0110 \\
\hline $61-80$ & 0.0411 & 0.0705 & 0.0610 & 0.0738 & 0.0925 & 0.1249 & 0.0064 & 0.0089 & -0.0034 & 0.0236 & 0.0061 & -0.0243 & 0.0177 & 0.0100 & 0.0039 & 0.0096 & -0.0091 \\
\hline
\end{tabular}

reported (Garzón et alı, 2018; Visser et al., 2016a; Eugenio Iglesias et al., 2013; Chakravarty et al., 2013; Patenaude et al., 2011). More importantly, it also includes structures seldom or never before considered in MRI atlases and parcellation methods, such as GPe, GPi, VTA, $3 \mathrm{~V}, 4 \mathrm{~V}$, ic, fx, PAG, PPN, Cl. The technique handles structures of varying sizes well, as indicated by dilated overlap and boundary distance. Additional structures can be added, if they can be reliably delineated by expert raters on single-subject MRI at achievable resolutions. Some enhancement techniques such as building a multi-subject template (Pauli et al., 2018) or adding a denoising step (Bazin et al., 2019) may be beneficial. Co-registration to a high-precision atlas as in Ewert et al., 2018 may also improve the initial alignment over the MASSP group average template.

Age biases are present both in expert manual delineations and automated parcellation techniques. Age trajectories in volume and quantitative MR parameters indicate systematic shifts in contrast intensities and an increasing variability with age, associated with changing myelination, iron deposition, and brain atrophy (Draganski et al., 2011; Daugherty and Raz, 2013; Fjell et al., 2013; Keuken et al., 2017). These changes seem only to impact the parcellation accuracy for age groups beyond 60 years and age-matched priors did not provide specific improvements, thus indicating that an explicit modeling of age effects may be required to further improve parcellation quality in elderly populations. These results also point to exercising caution when applying automated parcellation methods to study morphometry in elderly or diseased populations, where measured differences may include biases. They also point out that while global volume and local thickness are indeed affected by such biases, quantitative MRI measures are much more robust. Note that this bias is likely present is many automated methods, although they have not been systematically investigated due to the extensive manual labor required. Interestingly, biases also exist in expert delineations: when the size or shape of a structure is refined in neuroanatomical studies, experts may become more or less conservative in their delineations. Automated methods provide a more objective measure in such case, as the source of their bias is explicitly encoded in the atlas prior delineations and computational model. Important applications of subcortical parcellation also include deep-brain stimulation surgery (Ewert et al., 2018), where the number of structures parcellated by MASSP can 
help neurosurgeons orient themselves more easily, although precise targeting will still require manual refinements, especially in neurodegenerative diseases.

We observed that dilated overlap, that is, the overlap of structures up to one voxel, provided a measure of accuracy largely independent of size, for automated or manual delineations. Imprecision in the range of one voxel in the boundary is to be expected due to partial voluming which impacts Dice overlap. The dilated overlap measure is a better representative of performance and indicates that conservative or inclusive versions of the subcortical regions can be obtained by eroding or dilating the estimated boundary by a single voxel. Such masks may be useful when separating functional MRI signals between neighboring nuclei or when locating smaller features inside a structure. Additionally, the Bayesian estimation framework provides voxel-wise probability values, which can also be used to further weight the contribution of each voxel within a region in subsequent analyses.

In summary, our method provides fast and accurate parcellation for subcortical structures of varying size, taking advantage of the high resolution offered by $7 \mathrm{~T}$ and the specificity of quantitative MRI. The algorithm is based on an explicit model of structures given in a Bayesian framework and is free of tuning parameters. Given a different set of regions of interest or different populations, new priors can be automatically generated and used as the basis for the algorithm. If more MRI contrasts are available, the method can also be augmented to take them into account. The main requirement for the technique is a set of manual delineations of all the structures of interest in a small group of representative subjects. Performance may further improve with the number of included structures, as the number of distinct interfaces increases, refining in particular the intensity priors. In future works, we plan to include more structures or sub-structures and model the effects of age on the priors. We hope that the method, available in open source, will help neuroscience researchers to include more subcortical regions in their structural and functional imaging studies.

\section{Materials and methods}

\section{Data acquisition}

Our parcellation method has been developed for the MP2RAGEME sequence (Caan et alı, 2019). Briefly, the MP2RAGEME consists of two interleaved MPRAGEs with different inversions and four echoes in the second inversion. Based on these images, one can estimate quantitative MR parameters of R1, R2* and QSM. In this work, we used the following sequence parameters: inversion times $\mathrm{TI1}, 2=670 \mathrm{~ms}, 3675.4 \mathrm{~ms}$; echo times TE1 = $3 \mathrm{~ms}$, TE2,1-4 = 3, 11.5, 19, $28.5 \mathrm{~ms}$; flip angles FA1,2 $=4^{\circ}, 4^{\circ} ;$ TRGRE1,2 = $6.2 \mathrm{~ms}, 31 \mathrm{~ms} ;$ bandwidth $=404.9 \mathrm{MHz}$; TRMP2RAGE = $6778 \mathrm{~ms}$; SENSE acceleration factor $=2 ; \mathrm{FOV}=205 \times 205 \times 164 \mathrm{~mm}$; acquired voxel size $=0.70 \times 0.7 \times 0.7 \mathrm{~mm}$; acquisition matrix was $292 \times 290$; reconstructed voxel size $=0.64 \times 0.64 \times 0.7 \mathrm{~mm}$; turbo factor $(\mathrm{TFE})=150$ resulting in 176 shots; total acquisition time $=19.53 \mathrm{~min}$.

T1-maps were computed using a look-up table (Marques et al., 2010). T2*-maps were computed by least-squares fitting of the exponential signal decay over the multi-echo images of the second inversion. R1 and R2* maps were obtained as the inverse of T1 and T2*. For OSM, phase maps were pre-processed using iHARPERELLA (integrated phase unwrapping and background phase removal using the Laplacian) of which the OSM images were computed using LSQR (Li et al., 2014). Skull information was removed through creation of a binary mask using FSL's brain extraction tool on the reconstructed uniform T1-weighted image and then applied to the quantitative contrasts (Smith, 2002). As all images were acquired as part of a single sequence, no co-registration of the quantitative maps was required (see Figure 9).

\section{Anatomical structure delineations}

Manual delineations of subcortical structures were performed by two raters trained by an expert anatomist, according to protocols optimized to use the better contrast or combination of contrasts for each structure and to ensure a consistent approach across raters. The following 17 structures were defined on a group of 10 subjects (average age 24.4, eight female): striatum (Str), thalamus (Tha), lateral, 3rd and 4th ventricles (LV, 3V, 4V), amygdala (Amg), globus pallidus internal segment (GPi) and external segment (GPe), SN, STN, red nucleus (RN), ventral tegmental area (VTA), fornix $(\mathrm{fx})$, internal capsule (ic), periaqueductal gray (PAG), pedunculopontine nucleus (PPN), and claustrum $(\mathrm{Cl})$. Separate masks for left and right hemisphere were delineated except for $3 \mathrm{~V}, 4 \mathrm{~V}$, and $\mathrm{fx}$. In the 


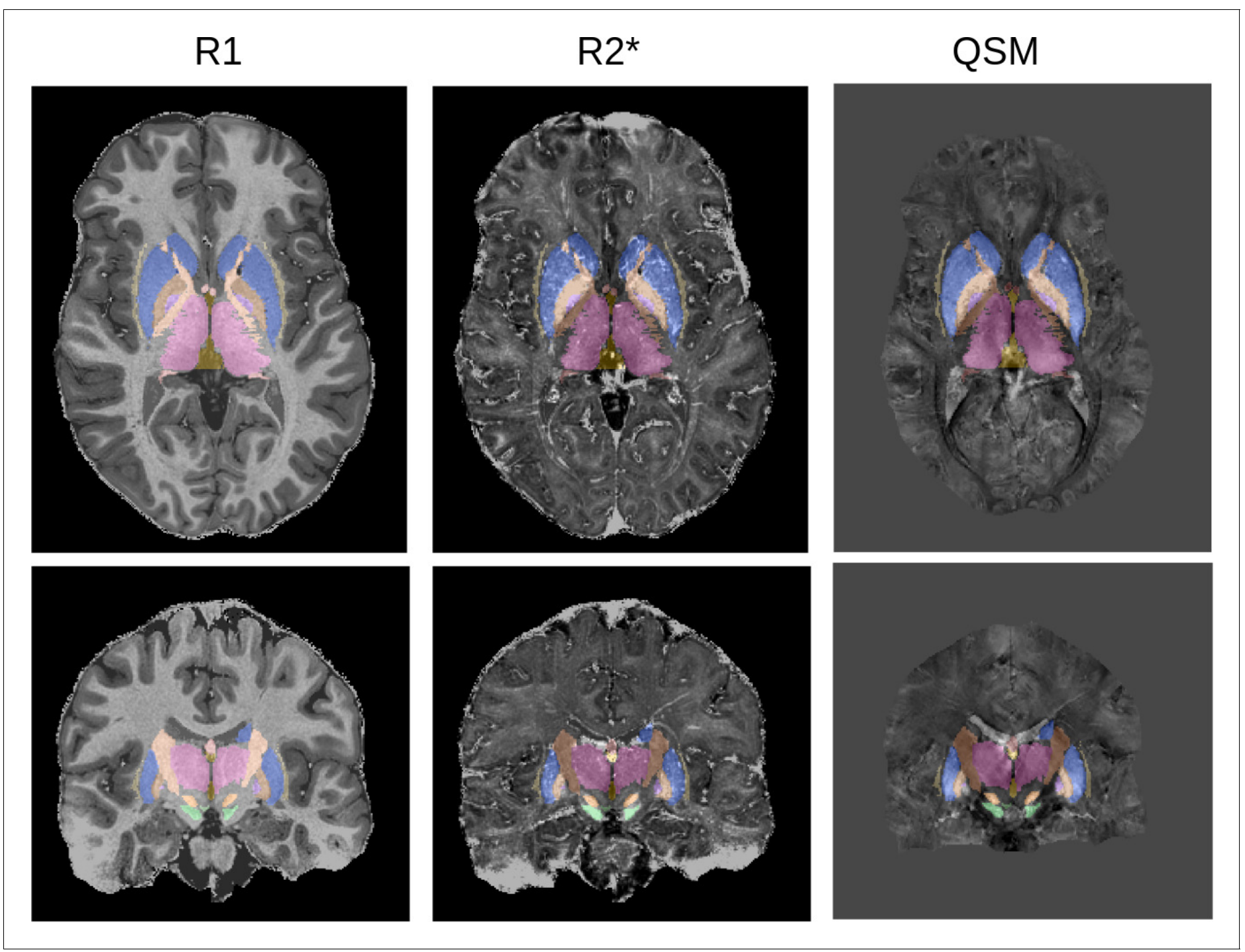

Figure 9. MP2RAGEME maps and delineations: quantitative R1 (left), quantitative R2* (middle), QSM (right). Manual delineations for the 17 structures of interest are overlaid on all images.

following the algorithm treats each side separately, resulting in a total of 31 distinct structures (see Figure 1).

\section{Anatomical interface priors}

In order to inform the algorithm, we built a series of priors derived from the manual delineations. Each subject was first co-registered to a MP2RAGEME anatomical template built from 105 subjects co-aligned with the MNI2009b atlas (Fonov et alo, 2011) with the SyN algorithm of ANTs (Avants et al., 2008) using successively rigid, affine, and non-linear transformations, high levels of regularization as recommended for the subcortex (Ewert et al., 2019) and mutual information as cost function.

The first computed prior is a prior of anatomical interfaces, recording the most likely location of boundaries between the different structures, defined as follows. Given two delineated structures $i, j$, let $\varphi_{i}, \varphi_{j}$ be the signed distance functions to their respective boundary, that is, $\varphi_{i}(x)$ is the Euclidean distance of any given voxel to the boundary of $i$, with a negative sign inside the structure. Then we define the interface $B_{i \mid j}$ with the distance function $d_{i \mid j}$ :

$$
d_{i \mid j}(x)=\min \left(\varphi_{i}(x), \varphi_{j}(x)-\delta, 0\right)
$$


where $\delta$ is a scale parameter for the thickness of the interface. These interfaces functions are not symmetrical, as the intensity inside $i$ next to $j$ is generally different from the intensity inside $j$ next to $i$. Based on this definition, the prior for a given interface based on $N$ manual delineations is given by:

$$
\begin{gathered}
P\left(x \in B_{i \mid j}\right) \sim \frac{1}{\sqrt{2 \pi \sigma_{i j}^{2}(x)}} \exp -\frac{1}{2} \frac{\mu_{i \mid j}^{2}(x)}{\sigma_{i j}^{2}(x)} \\
\mu_{i \mid j}(x)=\frac{1}{N} \sum_{n \in N} d_{i \mid j, n}(x), \sigma_{i \mid j}(x)=\sqrt{\frac{1}{N} \sum_{n \in N}\left(d_{i \mid j, n}(x)-\mu_{i \mid j}(x)\right)^{2}}+\delta
\end{gathered}
$$

These probability functions are calculated for all possible configurations including $i \mid i$, which represent the inside of each structure. We thus have a total of $N^{2}$ functions, but only a few are non-zero at a given voxel $x$, and we may keep only the 16 largest values to account for any number of interfaces in 3D (Bazin et al., 2007). Finally, we need to scale the prior to be globally consistent with the priors below by assuming that the 95th percentile of the highest kept $P\left(x \in B_{i \mid j}\right)$ values have a probability of 0.95. The scale parameter $\delta$ is set to one voxel, representing the expected amount of partial voluming. The resulting interface prior is shown in Figure 10A.

\section{Anatomical skeleton priors}

Next, we defined priors for the skeleton of each structure, representing their essential shape regardless of exact boundaries (Blum, 1973). As we are mostly interested in the most likely components of the skeleton or medial axis $S_{i}$, we follow a simple method to estimate its location:
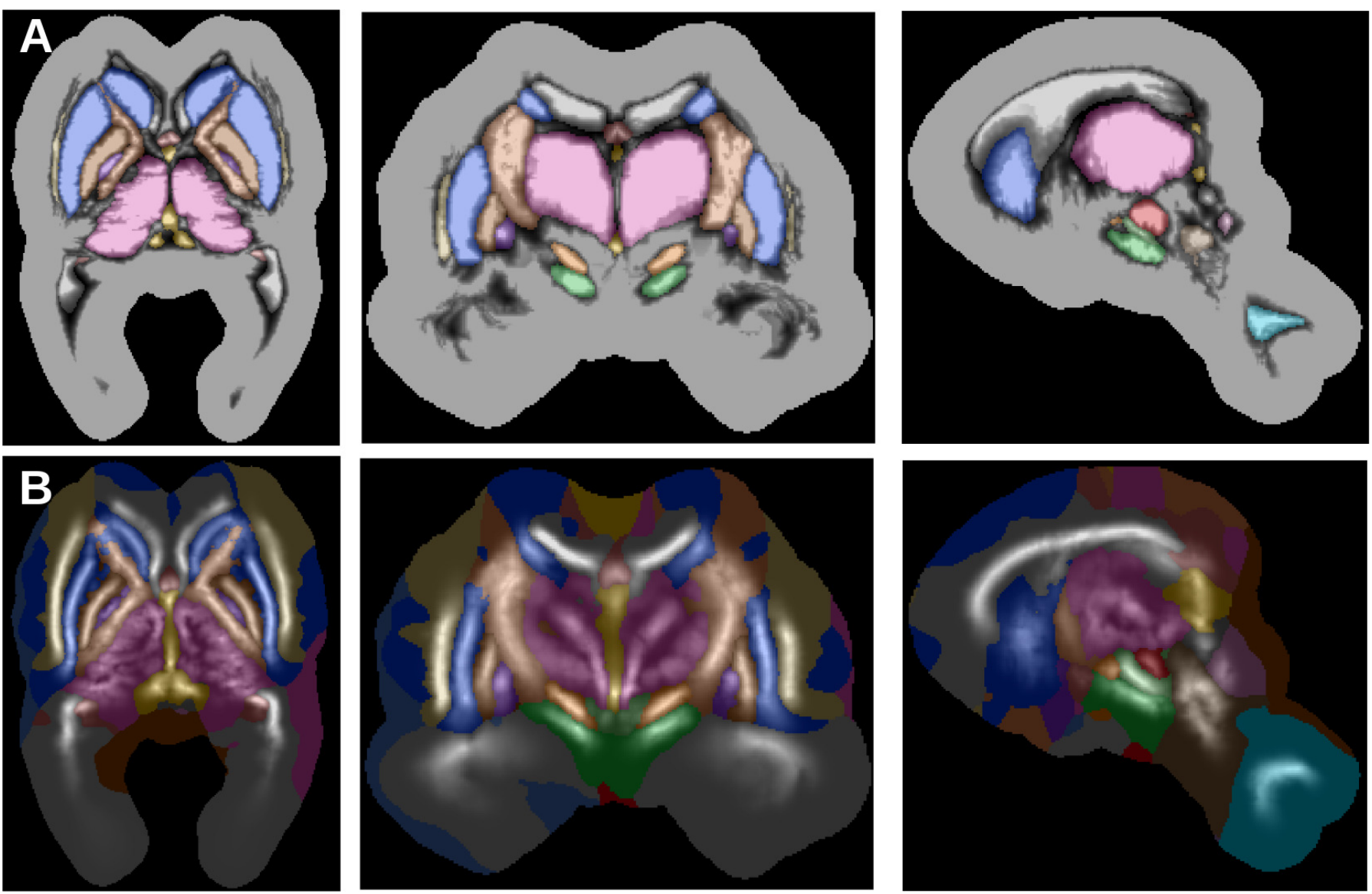

Figure 10. Anatomical interface (A) and skeleton (B) priors derived from the 10 manually delineated subjects. 


$$
S_{i}=\left\{x,\left|\nabla \varphi_{i}(x)\right|<\frac{1}{2}\right\}
$$

We define as $s_{i}(x)$ the signed distance function of this discrete skeleton, and define prior probabilities as above:

$$
\begin{gathered}
P\left(x \in S_{i}\right) \sim \frac{1}{\sqrt{2 \pi \sigma_{i}^{2}(x)}} \exp -\frac{1}{2} \frac{\mu_{i}^{2}(x)}{\sigma_{i}^{2}(x)} \\
\mu_{i}(x)=\frac{1}{N} \sum_{n \in N} s_{i, n}(x) \sigma_{i}(x)=\sqrt{\frac{1}{N} \sum_{n \in N}\left(s_{i, n}(x)-\mu_{i}(x)\right)^{2}}+\delta
\end{gathered}
$$

The skeletons are defined inside each structure, which implies $P\left(x \in S_{i}\right) \leq P\left(x \in B_{i \mid i}\right)$. To respect this relationship, we scale $P\left(x \in S_{i}\right)$ with the same factor as $P\left(x \in B_{i \mid i}\right)$ but use $\sqrt{P\left(x \in S_{i}\right)}$ when combining probabilities during the estimation stage. The obtained anatomical skeleton priors are given on Figure 10B.

\section{Interface intensity priors}

While anatomical priors already provide rich information, they are largely independent of the underlying MRI. From the co-aligned quantitative MRI maps and manual delineations, we defined intensity priors for every interface $i \mid j$, in the form of intensity histograms to ensure a flexible representation of intensity distributions. Given a quantitative contrast $R_{n}(x)$, we built a histogram $H_{i \mid j, n}$ for each subject $n$ and interface $i \mid j$. Histograms have 200 bins covering the entire intensity range within a radius of 10 $\mathrm{mm}$ from any of the delineated structures. To obtain an average histogram, we combine each histogram with a weighting function $w_{n}(x)$ giving the likelihood of the subject's intensity measurement compared to the group:

$$
w_{i \mid j, n}(x)=P\left(x \in B_{i \mid j}\right) \frac{1}{\sqrt{2 \pi \sigma_{R}^{2}}} \exp -\frac{1}{2} \frac{\left(R_{n}(x)-\mu_{R}(x)\right)^{2}}{\sigma_{R}(x)^{2}}
$$

where $\mu_{R}(x)$ is the median of the $R_{n}(x)$ values at $x$, and $\sigma_{R}(x)$ is 1.349 times the inter-quartile range of $R_{n}(x)$. These are robust estimators of the mean and standard deviation, used here to avoid biases by intensity outliers. To further combine the R1, R2* , and QSM contrasts we take the geometric mean of the histogram probabilities: $H_{i \mid j}(x)=\prod_{R} H_{i \mid j}(R(x))^{1 / 3}$.

\section{Global volume priors}

The last type of priors extracted from manual delineations are volume priors for each of the structure. Here, we assume a log-normal distribution for the volumes $V_{i}$ and simply estimate the mean $\mu_{V, i}$ and standard deviation $\sigma_{V, i}$ of $\log V_{i, n}$ over the subjects.

\section{Voxel-wise posterior probabilities}

When parcellating a new subject, we first co-register its R1, R2* , and QSM maps jointly to the template and use the inverse transformation to deform the anatomical priors into subject space. Then we derive voxel-wise posteriors as follows:

$$
\begin{gathered}
P\left(x \in B_{i \mid j} \mid R(x)\right) \sim P\left(x \in B_{i \mid j}\right) H_{i \mid j}(x) \text { if } i \neq j \\
\text { and } \\
P\left(x \in B_{i \mid i} \mid S_{i}(x), R(x)\right) \sim \max \left(P\left(x \in B_{i \mid i}\right), P\left(x \in S_{i}\right)^{1 / 2}\right) H_{i \mid i}(x)
\end{gathered}
$$

Once again we should compute all possible combinations, but due to the multiplication of the priors we can restrict ourselves to the 16 highest probabilities previously estimated. To balance the contribution of the anatomical priors and the intensity histograms, we also need to normalize the intensity priors sampled on the subject's intensities. We use the same approach, namely assuming that the 95th percentile of the highest kept $H_{i \mid j}(x)$ values have a probability of 0.95 , separately for each contrast. The voxel-wise parcellation and posteriors obtained are shown in Figure 11A. 

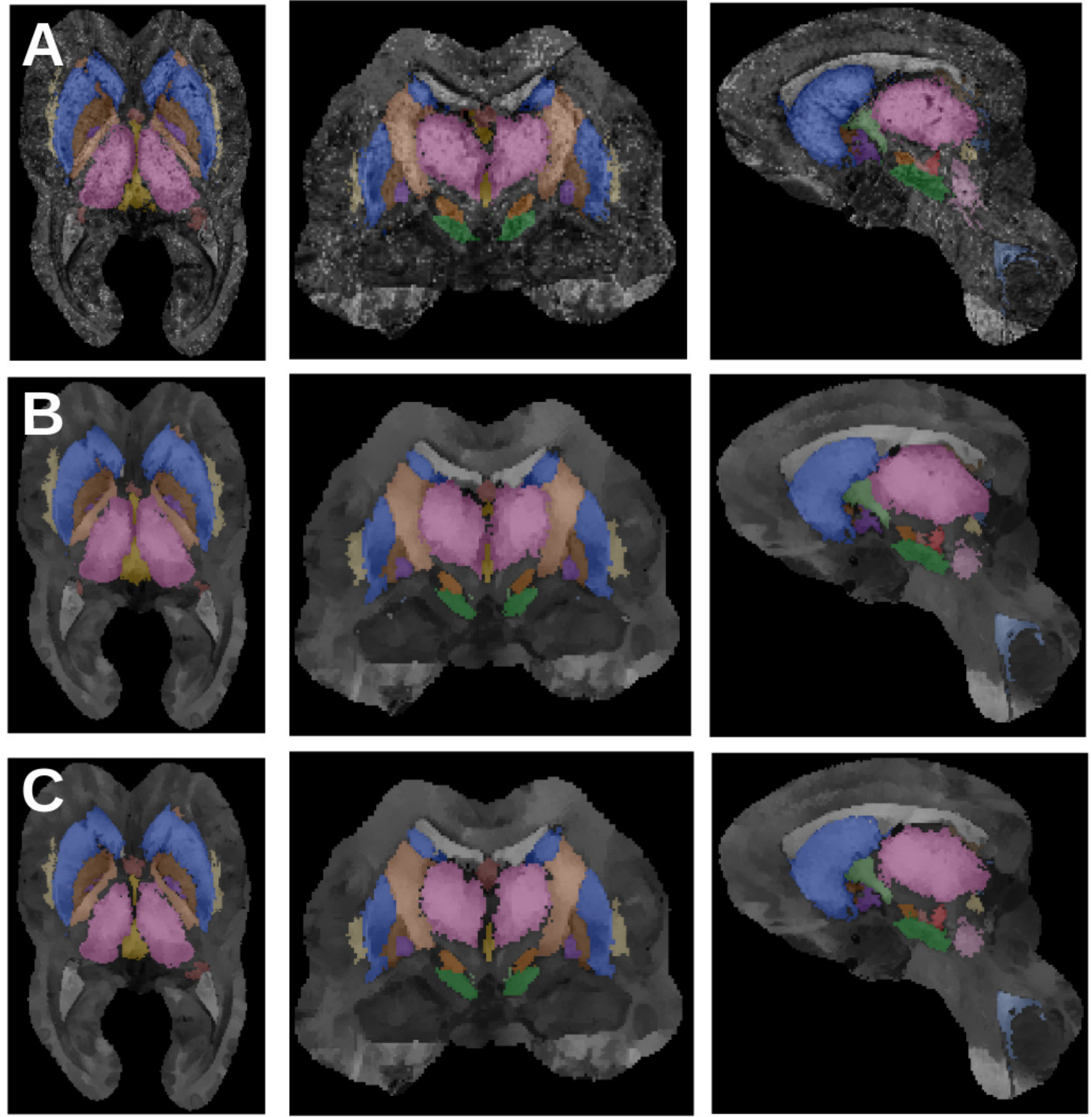

Figure 11. Successive parcellation results: (A) voxel-wise posteriors and parcellation, (B) diffused posteriors and parcellation, (C) topology-corrected posteriors and final region-growing parcellation.

\section{Markovian diffusion}

The voxel-wise posteriors are independent from each other and do not reflect the continuous nature of the structures. The next step is to combine information from neighboring voxels. We define a sparse Markov Random Field model for the posteriors: 


$$
P\left(x \in B_{i \mid j} \mid R, S, C\right)=\sum_{y \in C(x)} P(y \sim x \mid R) P\left(y \in B_{i \mid j} \mid R, S, C\right)
$$

with $P(y \sim x \mid R)=\prod_{R} \exp -(R(y)-R(x))^{2} / 2 \sigma_{R}^{2}$, where $\sigma_{R}$ is the median of the standard deviations $\sigma_{i \mid j, R}$ of the contrast histograms $H_{i j}(R(x))$. The neighborhood $C(x)$ is defined as $x$ itself and the four 26connected neighboring voxels with highest probability $P(y \sim x \mid R)$, thus representing the neighbors most likely to be connected to $x$. The model is similar to a diffusion process and can be estimated with an iterated conditional modes (ICM) approach, updating sequentially the probabilities (Bazin and Pham, 2007):

$$
P\left(x \in B_{i \mid j} \mid R, S, C\right) \leftarrow \sum_{y \in C(x)} P(y \sim x \mid R) P\left(y \in B_{i \mid j} \mid R, S, C\right)
$$

from the initial voxel-wise posteriors until the ratio of changed parcellation labels decreases below 0.1 , typically within $50-80$ iterations. The diffused probabilities and parcellation are shown in Figure 11B.

\section{Topology correction}

The final step of the parcellation algorithm takes a global view of the individual structures, growing from the highest posterior values inside toward the boundaries. This region growing approach makes the implicit assumption that posterior maps should be monotonically decreasing from inside to outside, which is not necessarily the case. Therefore, we perform first a topology correction step on the individual structure posteriors $P(x \in i \mid R, B, S, C)=\max _{i \mid j} P\left(x \in B_{i \mid j} \mid R, S, C\right)$ with a fast marching algorithm (Bazin and Pham, 2007). While the corrected posterior is very similar to the original one (see Figure 11C), it ensures that all regions obtained by growing to a threshold have spherical object topology.

\section{Anatomical region growing}

Last, we turn the posteriors into optimized parcellations, by growing them concurrently (to avoid overlaps) until the target volume for each structure is reached. Given the volume $V_{i}(R, B, S, C)$ of the parcellation of the diffused and topology-corrected posteriors, we define the following target volume:

$$
\hat{V}_{i}=P\left(V_{i} \mid \mu_{V, i}, \sigma_{V, i}\right) V_{i}+\left(1-P\left(V_{i} \mid \mu_{V, i}, \sigma_{V, i}\right)\right) \exp \mu_{V, i}
$$

taking a weighted average of the volume estimated from the data and the prior volume. This approach ensures that even in extreme cases where some structures have low posteriors, they are still able to grow to a plausible size. The region growing algorithm is driven from the most likely voxels, defined as $P(x \in i \mid R, B, S, C)-\max _{j \neq i} P(x \in j \mid R, B, S, C)$, and further modulated to follow isocontours of the skeleton prior:

$$
\begin{gathered}
P(x \leftarrow y) \sim P(y \in i \mid R, B, S, C)-\max _{j \neq i} P(y \in j \mid R, B, S, C) \\
-\left|P\left(y \in S_{i}\right)-P\left(x \in S_{i}\right)\right|
\end{gathered}
$$

Directionality of internal structures is a useful tool for understanding mechanical function in bones (Maquer et al., 2015). Here, we adapt this concept by using the skeleton isocontours as a representation of internal directionality, maintaining the intrinsic shape of structures. Thus, voxels with highest probability compared to the other structures and with similar distance to the internal skeleton are preferentially selected. The final parcellation is given in Figure $11 \mathrm{C}$.

\section{Validation metrics}

To validate the method against manual expert delineations, we compared the MASSP results and the expert delineations with the following three measures:

1. The Dice overlap coefficient (Dice, 1945) $D(A, B)=\frac{2 A \cap B}{A+B}$, which measures the strict overlap between voxels in both delineation; 
2. The dilated overlap coefficient $d D(A, B)=\frac{A \cup d(B)+B \cup d(A)}{A+B}$, where $d($.$) is a dilation of the delineation$ by one voxel, which measures the overlap between delineations allowing for one voxel of uncertainty;

3. The average surface distance $\operatorname{asd}(A, B)$, measuring the average distance between voxels on the surface boundary of the first delineation to the other one and reciprocally, which measures the distance between both delineations.

We computed all three measures for the manual delineations from the two independent raters, as well as the ratio of overlaps (automated over manual) and distances (manual over automated) to compare both performances, as detailed in the Results section.

\section{Comparisons with other automated methods}

To assess the performance of MASSP compared to existing parcellation tools, we ran Freesurfer (Fischl et al., 2002), FSL FIRST (Patenaude et alı, 2011) and a multi-atlas registration approach (coregistering 9 of the 10 manually delineated subjects on the remaining one with ANTs [Avants et al., 2008] and labeling each structure by majority voting, similarly to the MAGeT Brain approach of Chakravarty et al., 2013). Freesurfer and FIRST were run on the skull-stripped R1 map, while the multi-atlas approach used all three R1, R2*, and OSM contrasts. All methods were compared in terms of Dice overlap, dilated overlap and average surface distance. We also assessed the presence of a systematic volume bias, defined as the average of the signed difference of the estimated structure volume to the manually delineated volume, normalized by the manually delineated volume.

\section{Application to new MRI contrasts}

Before applying MASSP to unseen contrasts, we need to convert its intensity prior histograms $H_{i \mid j, R}$ to the new intensities. In order to perform this mapping, we first created a groupwise median of the HCP subjects, by co-registering every subject to the MASSP template using ANTs with non-linear registration and both $\mathrm{T} 1 \mathrm{w}, \mathrm{T} 2 \mathrm{w}$ contrasts matched to the template's $\mathrm{R} 1$ and R2* maps. The histogram bins are then updated as follows:

$$
H_{\mathrm{bin}, i \mid j, R} \equiv \sum_{x \mid R(x) \in \operatorname{bin}} P\left(x \in B_{i \mid j}\right) H_{i \mid j, R 1} H_{i \mid j, R 2 *} H_{i \mid j, Q S M}
$$

adding the joint probability of the quantitative contrasts weighted by their importance for each interface to define the new intensity histograms. This model is essentially projecting the joint likelihood of the MASSP contrasts onto the new contrasts, assuming that the co-registration between the two is accurate enough. With these new histograms, we compared the test-retest reliability and overall agreement of MASSP with Freesurfer parcellations included in the HCP pre-processed data set.

\section{Measurement of structure thickness}

Finally, when comparing derived measures obtained over the lifespan with MASSP compared to manual delineations, we explored the utility of a shape thickness metric, based on the medial representation. Given the signed distance function $\varphi_{i}$ of the structure boundary and $s_{i}$ of the structure skeleton, the thickness is given by:

$$
t h_{i}(x)=2\left(s_{i}(x)-\varphi_{i}(x)\right)
$$

Like in cortical morphometry, thickness is a local measure, defined everywhere inside the structure, and expected to provide additional information about anatomical variations. Indeed, a similar measure of shape thickness has recently been able to highlight subtle anatomical changes in depression (Ho et al., 2020).

\section{Software implementation}

The proposed method, Multi-contrast Anatomical Subcortical Structure Parcellation (MASSP), has been implemented as part of the Nighres toolbox (Huntenburg et al., 2018), using Python and Java for optimized processing. The software is available in open source from (release-1.3.0) and . A 
complete parcellation pipeline is included with the Nighres examples. Computations take under 30 min per subject on a modern workstation.

\section{Acknowledgements}

We thank Josephine Groot, Nikita Berendonk, Nicky Lute for their help collecting the AHEAD database, and Wietske van der Zwaag and Matthan Caan for their help in setting up the MP2RAGEME sequence. We also thank Steven Miletić and Dagmar Timmann for stimulating discussions around this topic, and all undergraduate students who contributed to the manual delineations. This work was supported by a NWO Vici grant (BF) and a NWO STW grant (AA, BF). HCP data were provided by the Human Connectome Project, WU-Minn Consortium (Principal Investigators: David Van Essen and Kamil Ugurbil; 1U54MH091657) funded by the $16 \mathrm{NIH}$ Institutes and Centers that support the $\mathrm{NIH}$ Blueprint for Neuroscience Research; and by the McDonnell Center for Systems Neuroscience at Washington University.

\section{Additional information}

Funding

\begin{tabular}{lll} 
Funder & Grant reference number & Author \\
\hline $\begin{array}{ll}\text { Nederlandse Organisatie voor } \\
\text { Wetenschappelijk Onderzoek }\end{array}$ & Birte U Forstmann \\
\hline $\begin{array}{ll}\text { Nederlandse Organisatie voor } \\
\text { Wetenschappelijk Onderzoek }\end{array}$ & STW & Anneke Alkemade \\
\hline
\end{tabular}

The funders had no role in study design, data collection and interpretation, or the decision to submit the work for publication.

Author contributions

Pierre-Louis Bazin, Conceptualization, Software, Formal analysis, Validation, Investigation, Visualization, Methodology, Writing - original draft; Anneke Alkemade, Data curation, Formal analysis, Validation, Investigation, Methodology, Writing - review and editing; Martijn J Mulder, Resources, Data curation, Software, Methodology, Writing - review and editing; Amanda G Henry, Conceptualization, Methodology, Writing - review and editing; Birte U Forstmann, Conceptualization, Resources, Formal analysis, Supervision, Funding acquisition, Project administration, Writing - review and editing

Author ORCIDs

Pierre-Louis Bazin (iD https://orcid.org/0000-0002-0141-5510

Anneke Alkemade (DiD http://orcid.org/0000-0002-3234-353X

Amanda G Henry (iD http://orcid.org/0000-0002-2923-4199

Birte U Forstmann (iD http://orcid.org/0000-0002-1005-1675

Ethics

Human subjects: Informed consent and consent to publish, including consent to publish anonymized imaging data, was obtained for all subjects. Ethical approval was obtained with the University of Amsterdam Faculty of Social and Behavioral Sciences LAB Ethics Review Board, with ERB number 2016-DP-6897.

Decision letter and Author response

Decision letter https://doi.org/10.7554/eLife.59430.sa1

Author response https://doi.org/10.7554/eLife.59430.sa2 


\section{Additional files}

Supplementary files

- Transparent reporting form

Data availability

The tool presented in this article is available in open source on Github (https://github.com/nighres/ nighres). The atlases necessary to run the algorithm have been deposited on the University of Amsterdam FigShare (https://doi.org/10.21942/uva.12074175.v1 and https://doi.org/10.21942/uva. 12301106.v2). A single sample subject data set has been deposited on the University of Amsterdam FigShare (https://doi.org/10.21942/uva.12280316.v2). All the measurements used to generate the figures included in the article have been deposited on the University of Amsterdam FigShare (https://doi.org/10.21942/uva.12452444.v1).

The following previously published datasets were used:

\begin{tabular}{|c|c|c|c|c|}
\hline Author(s) & Year & Dataset title & Dataset URL & $\begin{array}{l}\text { Database and } \\
\text { Identifier }\end{array}$ \\
\hline $\begin{array}{l}\text { Alkemade A, } \\
\text { Mulder MJ, Groot } \\
\text { JM, Isaacs BR, } \\
\text { van Berendonk N, } \\
\text { Lute N, Isherwood } \\
\text { SJ, Bazin P-L, } \\
\text { Forstmann BU }\end{array}$ & 2020 & $\begin{array}{l}\text { The Amsterdam Ultra-high field } \\
\text { adult lifespan database (AHEAD): A } \\
\text { freely available multimodal } 7 \text { Tesla } \\
\text { submillimeter magnetic resonance } \\
\text { imaging database }\end{array}$ & $\begin{array}{l}\text { https://doi.org/10.21942/ } \\
\text { uva.10007840.v1 }\end{array}$ & $\begin{array}{l}\text { FigShare / University } \\
\text { of Amsterdam / } \\
\text { Amsterdam University } \\
\text { of Applied Sciences, } \\
\text { 10.21942/uva.1000 } \\
7840 . v 1\end{array}$ \\
\hline $\begin{array}{l}\text { Van Essen DC, } \\
\text { Smith SM, Barch } \\
\text { DM, Behrens TEJ, }\end{array}$ & 2013 & $\begin{array}{l}\text { The WU-Minn Human Connectome } \\
\text { Project }\end{array}$ & $\begin{array}{l}\text { https://www.humancon- } \\
\text { nectome.org/study/hcp- } \\
\text { young-adult }\end{array}$ & $\begin{array}{l}\text { WU-Minn HCP Retest } \\
\text { Data, hcp-young- } \\
\text { adult }\end{array}$ \\
\hline
\end{tabular}

\section{References}

Ahsan RL, Allom R, Gousias IS, Habib H, Turkheimer FE, Free S, Lemieux L, Myers R, Duncan JS, Brooks DJ, Koepp MJ, Hammers A. 2007. Volumes, spatial extents and a probabilistic atlas of the human basal ganglia and thalamus. Neurolmage 38:261-270. DOI: https://doi.org/10.1016/j.neuroimage.2007.06.004, PMID: 17851093

Alkemade A, Mulder MJ, Groot JM, Isaacs BR, van Berendonk N, Lute N, Isherwood SJ, Bazin PL, Forstmann BU. 2020. The Amsterdam Ultra-high field adult lifespan database (AHEAD): A freely available multimodal 7 tesla submillimeter magnetic resonance imaging database. Neurolmage 221:117200. DOI: https://doi.org/10.1016/j. neuroimage.2020.117200, PMID: 32745682

Andersen HH, Johnsen KB, Moos T. 2014. Iron deposits in the chronically inflamed central nervous system and contributes to neurodegeneration. Cellular and Molecular Life Sciences 71:1607-1622. DOI: https://doi.org/10. 1007/s00018-013-1509-8, PMID: 24218010

Avants BB, Epstein CL, Grossman M, Gee JC. 2008. Symmetric diffeomorphic image registration with crosscorrelation: evaluating automated labeling of elderly and neurodegenerative brain. Medical Image Analysis 12 : 26-41. DOI: https://doi.org/10.1016/j.media.2007.06.004, PMID: 17659998

Bazin P-L, Ellingsen LM, Pham DL. 2007. Digital Homeomorphisms in Deformable Registration. In: Karssemeijer $\mathrm{N}$, Lelieveldt B (Eds). Information Processing in Medical Imaging. Berlin, Heidelberg: Springer Berlin Heidelberg. p. 211-222. DOI: https://doi.org/10.1007/978-3-540-73273-0_18

Bazin P-L, Plessis V, Fan AP, Villringer A, Gauthier CJ. 2016. Vessel segmentation from quantitative susceptibility maps for local oxygenation venography. 2016 IEEE 13th International Symposium on Biomedical Imaging (ISBI) 1135-1138. DOI: https://doi.org/10.1109/ISBI.2016.7493466

Bazin PL, Alkemade A, van der Zwaag W, Caan M, Mulder M, Forstmann BU. 2019. Denoising High-Field MultiDimensional MRI with local complex PCA. Frontiers in Neuroscience 13:66. DOI: https://doi.org/10.3389/fnins. 2019.01066, PMID: 31649500

Bazin PL, Pham DL. 2007. Topology correction of segmented medical images using a fast marching algorithm. Computer Methods and Programs in Biomedicine 88:182-190. DOI: https://doi.org/10.1016/j.cmpb.2007.08. 006, PMID: 17942182

Bazin PL, Pham DL. 2008. Homeomorphic brain image segmentation with topological and statistical atlases. Medical Image Analysis 12:616-625. DOI: https://doi.org/10.1016/j.media.2008.06.008, PMID: 18640069

Blum H. 1973. Biological shape and visual science. I. Journal of Theoretical Biology 38:205-287. DOI: https://doi. org/10.1016/0022-5193(73)90175-6, PMID: 4689997

Caan MWA, Bazin P, Marques JP, Hollander G, Dumoulin SO, Zwaag W. 2019. MP2RAGEME: T 1 , T 2 * , and QSM mapping in one sequence at 7 tesla. Human Brain Mapping 40:1786-1798. DOI: https://doi.org/10.1002/ hbm.24490, PMID: 30549128 
Chakravarty MM, Bertrand G, Hodge CP, Sadikot AF, Collins DL. 2006. The creation of a brain atlas for image guided neurosurgery using serial histological data. Neurolmage 30:359-376. DOI: https://doi.org/10.1016/j. neuroimage.2005.09.041, PMID: 16406816

Chakravarty MM, Steadman P, van Eede MC, Calcott RD, Gu V, Shaw P, Raznahan A, Collins DL, Lerch JP. 2013. Performing label-fusion-based segmentation using multiple automatically generated templates: maget brain: label fusion segmentation using automatically generated templates. Human Brain Mapping 34:2635-2654. DOI: https://doi.org/10.1002/hbm.22092, PMID: 22611030

Daugherty A, Raz N. 2013. Age-related differences in iron content of subcortical nuclei observed in vivo: a metaanalysis. Neurolmage 70:113-121. DOI: https://doi.org/10.1016/j.neuroimage.2012.12.040, PMID: 23277110

de Hollander G, Keuken MC, van der Zwaag W, Forstmann BU, Trampel R. 2017. Comparing functional MRI protocols for small, iron-rich basal ganglia nuclei such as the subthalamic nucleus at $7 \mathrm{~T}$ and $3 \mathrm{~T}$. Human Brain Mapping 38:3226-3248. DOl: https://doi.org/10.1002/hbm.23586, PMID: 28345164

Dice LR. 1945. Measures of the amount of ecologic association between species. Ecology 26:297-302. DOI: https://doi.org/10.2307/1932409

Draganski B, Ashburner J, Hutton C, Kherif F, Frackowiak RS, Helms G, Weiskopf N. 2011. Regional specificity of MRI contrast parameter changes in normal ageing revealed by voxel-based quantification (VBQ). Neurolmage 55:1423-1434. DOI: https://doi.org/10.1016/j.neuroimage.2011.01.052, PMID: 21277375

Eugenio Iglesias J, Rory Sabuncu M, Van Leemput K. 2013. A unified framework for cross-modality multi-atlas segmentation of brain MRI. Medical Image Analysis 17:1181-1191. DOI: https://doi.org/10.1016/j.media.2013. 08.001, PMID: 24001931

Ewert S, Plettig P, Li N, Chakravarty MM, Collins DL, Herrington TM, Kühn AA, Horn A. 2018. Toward defining deep brain stimulation targets in MNI space: a subcortical atlas based on multimodal MRI, histology and structural connectivity. Neurolmage 170:271-282. DOI: https://doi.org/10.1016/j. neuroimage.2017.05.015, PMID: 28536045

Ewert S, Horn A, Finkel F, Li N, Kühn AA, Herrington TM. 2019. Optimization and comparative evaluation of nonlinear deformation algorithms for atlas-based segmentation of DBS target nuclei. Neurolmage 184:586598. DOI: https://doi.org/10.1016/j.neuroimage.2018.09.061, PMID: 30267856

Fischl B, Salat DH, Busa E, Albert M, Dieterich M, Haselgrove C, van der Kouwe A, Killiany R, Kennedy D, Klaveness S, Montillo A, Makris N, Rosen B, Dale AM. 2002. Whole brain segmentation: automated labeling of neuroanatomical structures in the human brain. Neuron 33:341-355. DOI: https://doi.org/10.1016/s0896-6273 (02)00569-x, PMID: 11832223

Fjell AM, Westlye LT, Grydeland H, Amlien I, Espeseth T, Reinvang I, Raz N, Holland D, Dale AM, Walhovd KB, Alzheimer Disease Neuroimaging Initiative. 2013. Critical ages in the life course of the adult brain: nonlinear subcortical aging. Neurobiology of Aging 34:2239-2247. DOI: https://doi.org/10.1016/j.neurobiolaging.2013. 04.006, PMID: 23643484

Fonov V, Evans AC, Botteron K, Almli CR, McKinstry RC, Collins DL, Brain Development Cooperative Group. 2011. Unbiased average age-appropriate atlases for pediatric studies. Neurolmage 54:313-327. DOI: https:// doi.org/10.1016/j.neuroimage.2010.07.033, PMID: 20656036

Forstmann BU, Keuken MC, Schafer A, Bazin PL, Alkemade A, Turner R. 2014. Multi-modal ultra-high resolution structural 7-Tesla MRI data repository. Scientific Data 1:140050. DOI: https://doi.org/10.1038/sdata.2014.50, PMID: 25977801

Forstmann BU, de Hollander G, van Maanen L, Alkemade A, Keuken MC. 2016. Towards a mechanistic understanding of the human subcortex. Nature Reviews Neuroscience 18:57-65. DOI: https://doi.org/10.1038/ nrn.2016.163, PMID: 27974841

Forstmann BU, Isaacs BR, Temel Y. 2017. Ultra high field MRI-Guided deep brain stimulation. Trends in Biotechnology 35:904-907. DOI: https://doi.org/10.1016/j.tibtech.2017.06.010, PMID: 28941469

Frazier JA, Chiu S, Breeze JL, Makris N, Lange N, Kennedy DN, Herbert MR, Bent EK, Koneru VK, Dieterich ME, Hodge SM, Rauch SL, Grant PE, Cohen BM, Seidman LJ, Caviness VS, Biederman J. 2005. Structural brain magnetic resonance imaging of limbic and thalamic volumes in pediatric bipolar disorder. American Journal of Psychiatry 162:1256-1265. DOI: https://doi.org/10.1176/appi.ajp.162.7.1256, PMID: 15994707

Garzón B, Sitnikov R, Bäckman L, Kalpouzos G. 2018. Automated segmentation of midbrain structures with high iron content. Neurolmage 170:199-209. DOI: https://doi.org/10.1016/j.neuroimage.2017.06.016, PMID: 28602 813

Herting MM, Johnson C, Mills KL, Vijayakumar N, Dennison M, Liu C, Goddings AL, Dahl RE, Sowell ER, Whittle S, Allen NB, Tamnes CK. 2018. Development of subcortical volumes across adolescence in males and females: a multisample study of longitudinal changes. Neurolmage 172:194-205. DOI: https://doi.org/10.1016/j. neuroimage.2018.01.020, PMID: 29353072

Hill RA, Li AM, Grutzendler J. 2018. Lifelong cortical myelin plasticity and age-related degeneration in the live mammalian brain. Nature Neuroscience 21:683-695. DOI: https://doi.org/10.1038/s41593-018-0120-6, PMID: 2 9556031

Ho TC, Gutman B, Pozzi E, Grabe HJ, Hosten N, Wittfeld K, Völzke H, Baune B, Dannlowski U, Förster K, Grotegerd D, Redlich R, Jansen A, Kircher T, Krug A, Meinert S, Nenadic I, Opel N, Dinga R, Veltman DJ, et al. 2020. Subcortical shape alterations in major depressive disorder: findings from the ENIGMA major depressive disorder working group. Human Brain Mapping 1:e24988. DOl: https://doi.org/10.1002/hbm.24988

Huntenburg JM, Steele CJ, Bazin PL. 2018. Nighres: processing tools for high-resolution neuroimaging. GigaScience 7:giy082. DOI: https://doi.org/10.1093/gigascience/giy082, PMID: 29982501 
Iglesias JE, Insausti R, Lerma-Usabiaga G, Bocchetta M, Van Leemput K, Greve DN, van der Kouwe A, Fischl B, Caballero-Gaudes C, Paz-Alonso PM, Alzheimer's Disease Neuroimaging Initiative. 2018. A probabilistic atlas of the human thalamic nuclei combining ex vivo MRI and histology. Neurolmage 183:314-326. DOI: https://doi. org/10.1016/j.neuroimage.2018.08.012, PMID: 30121337

Ji JL, Spronk M, Kulkarni K, Repovš G, Anticevic A, Cole MW. 2019. Mapping the human brain's corticalsubcortical functional network organization. Neurolmage 185:35-57. DOI: https://doi.org/10.1016/j. neuroimage.2018.10.006, PMID: 30291974

Johansen-Berg H. 2013. Human connectomics - what will the future demand? Neurolmage 80:541-544. DOI: https://doi.org/10.1016/j.neuroimage.2013.05.082, PMID: 23727322

Keuken MC, Bazin PL, Schäfer A, Neumann J, Turner R, Forstmann BU. 2013. Ultra-high 7T MRI of structural agerelated changes of the subthalamic nucleus. Journal of Neuroscience 33:4896-4900. DOI: https://doi.org/10. 1523/JNEUROSCI.3241-12.2013, PMID: 23486960

Keuken MC, Bazin PL, Backhouse K, Beekhuizen S, Himmer L, Kandola A, Lafeber JJ, Prochazkova L, Trutti A, Schäfer A, Turner R, Forstmann BU. 2017. Effects of aging on $T_{1}, T_{2}{ }^{*}$, and $\mathrm{QSM}$ MRI values in the subcortex. Brain Structure \& Function 222:2487-2505. DOI: https://doi.org/10.1007/s00429-016-1352-4, PMID: 28168364

Koshiyama D, Fukunaga M, Okada N, Yamashita F, Yamamori H, Yasuda Y, Fujimoto M, Ohi K, Fujino H, Watanabe Y, Kasai K, Hashimoto R. 2018. Role of subcortical structures on cognitive and social function in schizophrenia. Scientific Reports 8:1183. DOI: https://doi.org/10.1038/s41598-017-18950-2, PMID: 29352126

Li W, Avram AV, Wu B, Xiao X, Liu C. 2014. Integrated Laplacian-based phase unwrapping and background phase removal for quantitative susceptibility mapping. NMR in Biomedicine 27:219-227. DOI: https://doi.org/ 10.1002/nbm.3056, PMID: 24357120

Makowski C, Béland S, Kostopoulos P, Bhagwat N, Devenyi GA, Malla AK, Joober R, Lepage M, Chakravarty MM. 2018. Evaluating accuracy of striatal, pallidal, and thalamic segmentation methods: comparing automated approaches to manual delineation. Neurolmage 170:182-198. DOl: https://doi.org/10.1016/j.neuroimage. 2017.02.069, PMID: 28259781

Maquer G, Musy SN, Wandel J, Gross T, Zysset PK. 2015. Bone volume fraction and fabric anisotropy are better determinants of trabecular bone stiffness than other morphological variables. Journal of Bone and Mineral Research 30:1000-1008. DOI: https://doi.org/10.1002/jbmr.2437, PMID: 25529534

Marquand AF, Haak KV, Beckmann CF. 2017. Functional corticostriatal connection topographies predict goal directed behaviour in humans. Nature Human Behaviour 1:0146. DOI: https://doi.org/10.1038/s41562-017. 0146, PMID: 28804783

Marques JP, Kober T, Krueger G, van der Zwaag W, Van de Moortele PF, Gruetter R. 2010. MP2RAGE, a self bias-field corrected sequence for improved segmentation and T1-mapping at high field. Neurolmage 49:12711281. DOI: https://doi.org/10.1016/j.neuroimage.2009.10.002, PMID: 19819338

McCoy RC, Wakefield J, Akey JM. 2017. Impacts of Neanderthal-Introgressed sequences on the landscape of human gene expression. Cell 168:916-927. DOI: https://doi.org/10.1016/j.cell.2017.01.038, PMID: 28235201

Miletić S, Bazin PL, Weiskopf N, van der Zwaag W, Forstmann BU, Trampel R. 2020. fMRI protocol optimization for simultaneously studying small subcortical and cortical Areas at 7T. Neurolmage 219:116992. DOI: https:// doi.org/10.1016/j.neuroimage.2020.116992, PMID: 32480037

Mosley PE, Smith D, Coyne T, Silburn P, Breakspear M, Perry A. 2018. The site of stimulation moderates neuropsychiatric symptoms after subthalamic deep brain stimulation for Parkinson's disease. Neurolmage: Clinical 18:996-1006. DOI: https://doi.org/10.1016/j.nicl.2018.03.009, PMID: 29876284

Patenaude B, Smith SM, Kennedy DN, Jenkinson M. 2011. A Bayesian model of shape and appearance for subcortical brain segmentation. Neurolmage 56:907-922. DOI: https://doi.org/10.1016/j.neuroimage.2011.02 046, PMID: 21352927

Pauli WM, Nili AN, Tyszka JM. 2018. A high-resolution probabilistic in vivo atlas of human subcortical brain nuclei. Scientific Data 5:180063. DOI: https://doi.org/10.1038/sdata.2018.63, PMID: 29664465

Qiu A, Brown T, Fischl B, Ma J, Miller MI. 2010. Atlas generation for subcortical and ventricular structures with its applications in shape analysis. IEEE Transactions on Image Processing 19:1539-1547. DOI: https://doi.org/10. 1109/TIP.2010.2042099, PMID: 20129863

Sitek KR, Gulban OF, Calabrese E, Johnson GA, Lage-Castellanos A, Moerel M, Ghosh SS, De Martino F. 2019. Mapping the human subcortical auditory system using histology. post mortem MRI and in vivo MRI at 7T. bioRxiv. DOI: https://doi.org/10.1101/568139

Smith SM. 2002. Fast robust automated brain extraction. Human Brain Mapping 17:143-155. DOI: https://doi. org/10.1002/hbm.10062, PMID: 12391568

Steiner H, Tseng KY. 2017. Handbook of Basal Ganglia Structure and Function. In: Steiner H, Tseng K (Eds). Handbook of Behavioral Neuroscience. 24 Elsevier. p. 1-1036.

Tardif CL, Gauthier CJ, Steele CJ, Bazin PL, Schäfer A, Schaefer A, Turner R, Villringer A. 2016. Advanced MRI techniques to improve our understanding of experience-induced neuroplasticity. Neurolmage 131:55-72. DOI: https://doi.org/10.1016/j.neuroimage.2015.08.047, PMID: 26318050

Turner R. 2019. Myelin and modeling: bootstrapping cortical microcircuits. Frontiers in Neural Circuits 13:34. DOI: https://doi.org/10.3389/fncir.2019.00034, PMID: 31133821

Van Essen DC, Smith SM, Barch DM, Behrens TE, Yacoub E, Ugurbil K, WU-Minn HCP Consortium. 2013. The WU-Minn human connectome project: an overview. Neurolmage 80:62-79. DOI: https://doi.org/10.1016/j. neuroimage.2013.05.041, PMID: 23684880 
Visser E, Keuken MC, Douaud G, Gaura V, Bachoud-Levi AC, Remy P, Forstmann BU, Jenkinson M. $2016 a$. Automatic segmentation of the striatum and globus pallidus using MIST: multimodal image segmentation tool. Neurolmage 125:479-497. DOI: https://doi.org/10.1016/j.neuroimage.2015.10.013, PMID: 26477650

Visser E, Keuken MC, Forstmann BU, Jenkinson M. 2016b. Automated segmentation of the Substantia Nigra, subthalamic nucleus and red nucleus in 7T data at young and old age. Neurolmage 139:324-336. DOI: https:// doi.org/10.1016/j.neuroimage.2016.06.039, PMID: 27349329

Wang BT, Poirier S, Guo T, Parrent AG, Peters TM, Khan AR, Styner MA, Angelini ED. 2016. Generation and evaluation of an ultra-high-field atlas with applications in DBS planning. Proceedings of SPIE Medical Imaging.

Weiskopf N, Suckling J, Williams G, Correia MM, Inkster B, Tait R, Ooi C, Bullmore ET, Lutti A. 2013. Quantitative multi-parameter mapping of $\mathrm{R} 1, \mathrm{PD}\left({ }^{*}\right), \mathrm{MT}$, and $\mathrm{R} 2\left(^{*}\right)$ at $3 \mathrm{~T}$ : a multi-center validation. Frontiers in Neuroscience 7:95. DOI: https://doi.org/10.3389/fnins.2013.00095, PMID: 23772204

Xiao Y, Fonov V, Bériault S, Al Subaie F, Chakravarty MM, Sadikot AF, Pike GB, Collins DL. 2015. Multi-contrast unbiased MRI atlas of a parkinson's disease population. International Journal of Computer Assisted Radiology and Surgery 10:329-341. DOI: https://doi.org/10.1007/s11548-014-1068-y, PMID: 24841147

Yeatman JD, Wandell BA, Mezer AA. 2014. Lifespan maturation and degeneration of human brain white matter. Nature Communications 5:4932. DOI: https://doi.org/10.1038/ncomms5932, PMID: 25230200

Yelnik J, Bardinet E, Dormont D, Malandain G, Ourselin S, Tandé D, Karachi C, Ayache N, Cornu P, Agid Y. 2007. A three-dimensional, histological and deformable atlas of the human basal ganglia. I. Atlas construction based on immunohistochemical and MRI data. Neurolmage 34:618-638. DOI: https://doi.org/10.1016/j. neuroimage.2006.09.026, PMID: 17110133

Zhang Y, Wei H, Cronin MJ, He N, Yan F, Liu C. 2018. Longitudinal atlas for normative human brain development and aging over the lifespan using quantitative susceptibility mapping. Neurolmage 171:176-189. DOI: https:// doi.org/10.1016/j.neuroimage.2018.01.008, PMID: 29325780 